\title{
CATEGORY OF VECTOR BUNDLES ON ALGEBRAIC CURVES AND INFINITE DIMENSIONAL GRASSMANNIANS*
}

\author{
Motohico Mulase** \\ Department of Mathematics \\ and \\ Institute of Theoretical Dynamics \\ University of California \\ Davis, CA 95616
}

\begin{abstract}
Equivalence between the following categories is established: 1) A category of arbitrary vector bundles on algebraic curves defined over a field of arbitrary characteristic, and 2) a category of infinite dimensional vector spaces corresponding to certain points of Grassmannians together with their stabilizers. Our contravariant functor between these categories gives a full generalization of the well-known Krichever map, which assigns points of Grassmannians to the geometric data consisting of curves and line bundles. As an application, a solution to the classical problem of Wallenberg-Schur of classifying all commutative algebras consisting of ordinary differential operators is obtained.
\end{abstract}

Table of Contents

0. Introduction . . . . . . . . . . . . . . . . . . . . . . . . . . 2

1. The Grassmannians and the category $\mathcal{S}(\nu)$. . . . . . . . . . . . . . . . 6

2. The category $\mathcal{Q}(\nu)$ of geometric data on algebraic curves . . . . . . . . 8

3. Categorical equivalence of $\mathcal{S}(\nu)$ and $\mathcal{Q}(\nu)$. . . . . . . . . . . . . . . . 16

4. Pseudo-differential operators acting on the Grassmannians . . . . . . . . 26

5. Classification of commuting ordinary differential operators . . . . . . . . 36

6. The KP flows on the quotients of the Grassmannians . . . . . . . . . . 40

7. Appendix - a theorem of M. Sato . . . . . . . . . . . . . . . . . . . 45

References . . . . . . . . . . . . . . . . . . 50

1991 Mathematics Subject Classification. Primary 14F05, 47E05, 58B15.

*Published in International Journal of Mathematics 1, (1990) 293-342.

**Research supported by NSF Grant DMS 8610730, The Institute for Advanced Study, and Aspen Center for Physics. 


\section{Introduction.}

In this paper, we give a complete solution to the classical problem of WallenbergSchur: determine all the commutative algebras consisting of ordinary differential operators.

In the 1903 paper [W], G. Wallenberg studied commuting pairs of ordinary differential operators. Let $P$ and $Q$ be ordinary differential operators. We say $P$ and $Q$ commute if

$$
[P, Q]=P \cdot Q-Q \cdot P=0 .
$$

He tried to determine all such commuting pairs. First, he did it for the case when ord $P=1$ and ord $Q=n$, and then the case of ord $P=$ ord $Q=2$. These are easy cases and he gave an explicit determination. Then he moved on to the nontrivial cases such as ord $P=2$ and ord $Q=3$. He discovered that the coefficients of $P$ and $Q$ are given by Weierstrass elliptic functions. Let us take

$$
\left\{\begin{array}{l}
P=\left(\frac{d}{d x}\right)^{2}-2 u(x) \\
Q=\left(\frac{d}{d x}\right)^{3}-3 u(x) \frac{d}{d x}-\frac{3}{2} u^{\prime}(x),
\end{array}\right.
$$

where $u^{\prime}(x)$ is the derivative of $u(x)$ with respect to $x$. It is an easy calculation to show that $P$ and $Q$ commute if and only if $u$ satisfies

$$
u^{\prime \prime \prime}=12 u u^{\prime} .
$$

We can integrate this equation twice to obtain

$$
\left(u^{\prime}\right)^{2}=4 u^{3}-g_{2} u-g_{3},
$$

where $g_{2}$ and $g_{3}$ are constants of integration. Therefore, $P$ and $Q$ commute if and only if their coefficients are given by the Weierstrass elliptic functions and their degenerations, since $g_{2}$ and $g_{3}$ in (0.2) can be arbitrary. If $u$ satisfies $(0.2)$, then $P$ and $Q$ satisfy a polynomial relation

$$
Q^{2}=P^{3}-\frac{g_{2}}{4} P-\frac{g_{3}}{4}
$$

which defines a plane cubic curve. Wallenberg did not seem to be interested in his own discovery of this mysterious connection between commuting differential operators and algebraic curves, presumably because he was interested only in the explicit determination.

Two years later, inspired by [W], I. Schur found a remarkable fact. Since he obtained his famous Schur's Lemma in the same year of 1905, I propose to call his result 
Schur's Other Lemma. Let $P$ be an arbitrary ordinary differential operator of order greater than zero, and let $B_{P}$ be the set of all differential operators which commute with $P$. Then $B_{P}$ is a commutative algebra.

Schur's proof is based on the $\mathcal{D}$-module theory of one dimension introduced by G. Floquet [F] in 1879 and the theory of pseudo-differential operators in one variable invented by S. Pincherle $[\mathrm{P}]$ in 1897. (For his proof and more detail of the history of the subject, see [M4].)

Schur's Other Lemma does not hold for other algebras such as matrix algebras and the algebra of partial differential operators. It is one of the characteristic features of the case of ordinary differential operators. Because of this result, it is more natural to consider commutative algebras of ordinary differential operators than to look only at two elements of such an algebra. Therefore, a more natural problem in this direction is to classify all possible commutative algebras consisting of ordinary differential operators.

This problem has been studied by many authors and in diverse context of motivations, including Burchnall-Chaundy [BC], Gelfand-Dikii [GD], Krichever [K], Mumford [Mum], Segal-Wilson [SW] and Verdier [V]. For a commutative algebra $B$ of ordinary differential operators, we define

$$
\text { rank } B=G . C . D .\{\text { ord } P \mid P \in B\} .
$$

Complete geometric classification of rank one algebras was established by Krichever and Mumford. Their result is, roughly speaking, that every rank one algebra $B$ is in one-to-one correspondence with a geometric triple $(C, p, \mathcal{L})$ consisting of an arbitrary algebraic curve $C$, a smooth point $p \in C$ and a generic line bundle $\mathcal{L}$ on $C$ of degree genus $(C)-1$. It is important to notice that their correspondence is essentially constructible in both directions. After their work, many attempts have been made to classify higher rank algebras. Unfortunately, the obvious idea of using the triple $(C, p, \mathcal{F})$ with a vector bundle $\mathcal{F}$ does not work, because $B$ has far more informations than a triple.

Then Verdier $[\mathrm{V}]$ proposed a classification in terms of parabolic structures and connections of vector bundles defined on curves. As far as I know, this is the only result so far obtained which covers all the higher rank cases. But this is not a natural generalization of the theorem of Krichever and Mumford. From both an analytic and an algebraic point of view, it is natural to identify two algebras of ordinary differential operators $B_{1}$ and $B_{2}$ if there is an invertible function $f$ such that $B_{1}=f \cdot B_{2} \cdot f^{-1}$. Krichever and Mumford used this identification in their classification. But the theorem of $[\mathrm{V}]$ dealing with the higher rank cases does not incorporate this identification. And also, the correspondence is constructible only in one direction, from algebra to geometry.

The solution we are proposing in this paper works for an arbitrary rank, is a natural generalization of the theorem of Krichever and Mumford, and is constructible in both ways. 
Classification Theorem. There is a bijective correspondence between the following two sets of objects:

Analytic object: A commutative algebra $B$ of ordinary differential operators with a monic element (i.e. an operator whose leading coefficient is 1 ). For an invertible function $f$, we identify the algebras $B$ and $f \cdot B \cdot f^{-1}$.

Geometric object: An isomorphism class of quintets $(C, p, \mathcal{F}, \pi, \phi)$ consisting of an arbitrary complete irreducible algebraic curve $C$ of genus, say $g$, a smooth point $p \in C$, an arbitrary semistable vector bundle $\mathcal{F}$ on $C$ of rank $r$ and a fixed degree $d=r(g-1)$ and having no nontrivial holomorphic global sections, a local covering $\pi$ of $C$ ramified at $p$, and a special kind of local trivialization $\phi$ of $\mathcal{F}$ near $p$.

Remark. All the vector bundles appearing in this correspondence are semistable. I do not know how to characterize strictly stable ones in terms of the commutative rings of ordinary differential operators.

The above theorem is obtained as a corollary of more elaborate theorem of categorical equivalence between a category of quintets and a category of infinite dimensional vector spaces. After Segal and Wilson $[\mathrm{SW}]$ discovered an injective map, now called the Krichever map, of the set of geometric objects consisting of quintuples $(C, p, \mathcal{L}, z, \phi)$ into the infinite dimensional Grassmannian introduced by M. Sato [S], where $\mathcal{L}$ is a line bundle of degree $g-1$ and $z$ is a local coordinate of $C$ around $z$, people started to generalize this map to the data consisting of vector bundles. For example, if one uses $(C, p, \mathcal{F}, z, \phi)$, then one can construct a map of these quintuples into the Grassmannian of vector valued functions. It is possible to give a one-to-one correspondence between this Grassmannian of vector valued functions and Sato's original Grassmannian of scalar valued functions, and hence one obtains a map of higher rank objects into the single Grassmannian. However, such a map can never be functorial.

In this paper, a contravariant functor is presented as a higher rank generalization of the Krichever map. Let $\mathcal{Q}$ be the category of quintets $(C, p, \mathcal{F}, \pi, \phi)$ consisting of arbitrary curves and vector bundles, with absolutely no restrictions on ranks and degrees. Our functor $\chi$ makes this category equivalent with the other category $\mathcal{S}$ (S stands for Schur) of pairs $(A, W)$, where $W$ is a vector subspace of $V=$ $k((z))$ satisfying certain Fredholm conditions (therefore, it is indeed a point of the Grassmannian of Fredholm operators), and $A$ is a nontrivial subring of $V$ which stabilizes $W$, i.e. $A W \subset W$. The correspondence is basically obtained in the following way:

(1) $A=$ the set of holomorphic functions on $C \backslash\{p\}$;

(2) $W=$ the set of holomorphic sections of $\mathcal{F}$ on $C \backslash\{p\}$.

For a pair $(A, W)$, we define

rank $A=$ G.C.D. $\{$ pole order of $a(z) \mid a(z) \in A\}$. 
It coincides with the rank of the corresponding vector bundle $\mathcal{F}$ and the rank of the algebra $B$ defined by $(0.4)$.

If $W$ is a point of the big cell of the special Grassmannian $G(0,-1)$ (see Section 1 for definition), then $W$ determines a unique pseudo-differential operator $S$ of order zero. This is the theorem of Sato discovered in 1981 [S]. Let us introduce an identification of the coordinate $z^{-1}$ with the differential operator $\frac{d}{d x}$, which is in a sense the Fourier transform of the operator. Then $A$ becomes a ring of pseudo-differential operators with constant coefficients. Now define

$$
B=S \cdot A \cdot S^{-1} .
$$

It turns out that the condition $A W \subset W$ is precisely equivalent to the condition that $B$ contains only differential operators. Therefore, our classification theorem immediately follows from the categorical equivalence. The reason why we need a functor instead of just a bijection is that, in due course, we have to consider isomorphism relations among the quintets and the pairs $(A, W)$. We will see in Section 4 that a morphism among the geometric objects corresponds to an action of a pseudo-differential operator on the Grassmannians.

Remark. It is possible to define a category of commutative algebras of ordinary differential operators in a natural way. But it does not become equivalent with the category of the geometric data we have.

The ring $V$ acts on $V$ itself by multiplication, hence it defines an infinitesimal action on the Grassmannians. Sato found that this action on $G(0,-1)$ is nothing but the system of Kadomtsev-Petviashvili equations (the KP system) through the correspondence between points of $G(0,-1)$ and pseudo-differential operators of order zero. Then I proved in [M1] and [M2] that every finite dimensional orbit of the KP action is isomorphic to a generalized Jacobian variety of an algebraic curve. Thus the KP system produces all line bundles.

Since we now have the correspondence between vector bundles and points of the Grassmannian, it is natural to ask if the KP system produces all the vector bundles on arbitrary algebraic curves. By using our theorem of categorical equivalence together with the recent theory of Hitchin $[\mathrm{H}]$ and Beauville-Narasimhan-Ramanan $[\mathrm{BNR}]$, we can answer this question affirmatively. Namely, we can show that the KP action (more precisely, the action of $V$ on the Grassmannians) produces all generic vector bundles.

The paper is organized as follow.

In Section 1, we define the Grassmannians labeled by the index of the Fredholm condition. The category $\mathcal{S}$ of pairs $(A, W)$ is also defined.

In Section 2, the category of quintets consisting of geometric data is defined. An explicit construction of $(A, W)$ out of a quintet is given.

The equivalence between these categories is proved in Section 3. We do this by giving an explicit construction of the geometric data from $(A, W)$. We use the technique of $[\mathrm{Mum}]$ and $[\mathrm{V}]$ in this section. 
In order to relate the theory of previous sections with ordinary differential operators, we have to introduce more morphisms into these categories. These morphisms are defined by actions of pseudo-differential operators on the Grassmannians. In this section, the functor of Section 3 is extended to these thickened categories. The equivalence still holds in this case.

The geometric classification theorem of commutative algebras of ordinary differential operators is proved in Section 5.

The KP system, i.e. the $V$ action on the Grassmannians, is defined in a coordinate free manner in Section 6 and its finite dimensional orbits are examined. It is shown that all generic vector bundles on an arbitrary curve of genus greater than one appear in a orbit of this action.

In Appendix, we give a proof of the theorem of Sato. Since we change the formulation of the theorem from the original one used in $[\mathrm{S}]$ and $[\mathrm{SN}]$, our correspondence becomes canonical and rather simpler.

I express my hearty gratitude to Professor Pierre Deligne for many stimulating discussions and invaluable suggestions. In particular, the idea of incorporating categorical point of view was suggested by him. I am also grateful to Professor Goro Shimura for his important and useful comments made on the earlier version of this paper.

\section{The Grassmannians and the category $\mathcal{S}(\nu)$.}

Throughout this paper, we work with a field $k$ of an arbitrary characteristic unless otherwise stated. In Section 1, we define the infinite dimensional Grassmannian of index $\mu$ and level $\nu$ for every integers $\mu$ and $\nu$. Then we introduce a category of points of these Grassmannians.

Let $V=k((z))$ be the set of all formal Laurent series in one variable $z$. This is the field of quotients of the ring $k[[z]]$ of formal power series. There is a natural filtration

$$
\cdots \supset V^{(\nu+1)} \supset V^{(\nu)} \supset V^{(\nu-1)} \supset \cdots
$$

in $V$ defined by

$$
V^{(\nu)}=k[[z]] \cdot z^{-\nu}
$$

which is the set of all formal Laurent series whose pole order at $z=0$ is less than or equal to $\nu \in \mathbb{Z}$. We say $v \in V$ has order $\nu$ if $v \in V^{(\nu)} \backslash V^{(\nu-1)}$. Since

$$
\bigcup_{\nu \in \mathbb{Z}} V^{(\nu)}=V \quad \text { and } \bigcap_{\nu \in \mathbb{Z}} V^{(\nu)}=\{0\}
$$

the filtration (1.1) defines a natural complete topology in $V$ by defining $\left\{V^{(\nu)}\right\}_{\nu \in \mathbb{Z}}$ as a basis for open sets of $V$. For every vector subspace $W$ in $V$, let $\gamma(\nu)_{W}$ denote 
the natural map of $W$ into $V / V^{(\nu)}$ defined by

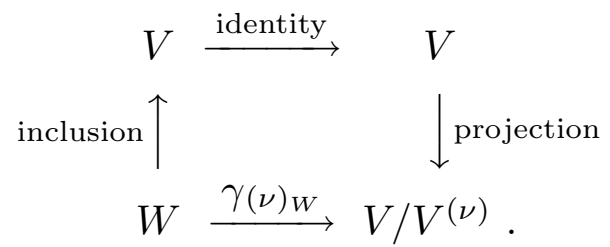

This map is said to be Fredholm if both of the kernel and cokernel of $\gamma(\nu)_{W}$ are of finite dimension. For a Fredholm map $\gamma$, we define the Fredholm index by Index $\gamma=\operatorname{dim}_{k} \operatorname{Ker} \gamma-\operatorname{dim}_{k} \operatorname{Coker} \gamma$.

Definition 1.1. For every integers $\mu, \nu \in \mathbb{Z}$, the following set is called the Grassmannian of index $\mu$ and level $\nu$ :

$$
G(\mu, \nu)=\left\{\text { closed vector subspace } W \mid \gamma(\nu)_{W} \text { is Fredholm of index } \mu\right\} .
$$

The big cell of the Grassmannian of index zero is defined by

$$
G^{+}(0, \nu)=\left\{W \in G(0, \nu) \mid \operatorname{Ker} \gamma(\nu)_{W}=\operatorname{Coker} \gamma(\nu)_{W}=0\right\} .
$$

Each $G(\mu, \nu)$ has a structure of infinite dimensional scheme by projective limit. Therefore, our Grassmannians are pro-algebraic varieties in the sense of Grothendieck. An element $v \in V$ of order $\nu^{\prime} \in \mathbb{Z}$ gives a continuous isomorphism $v: V \rightarrow V$ by multiplication. Thus $G(\mu, \nu)$ is always isomorphic to $G\left(\mu, \nu+\nu^{\prime}\right)$ for every $\mu$ and $\nu$. We note here that there is no canonical isomorphism between them.

Definition 1.2. Let $r$ be a positive integer, $\mu$ and $\nu$ be arbitrary integers. A pair $(A, W)$ is said to be a Schur pair of rank $r$, index $\mu$ and level $\nu$ if the following conditions are satisfied:

(1) $W$ is a point of the Grassmannian $G(\mu, \nu)$ of index $\mu$ and level $\nu$.

(2) $A \subset V$ is a $k$-subalgebra of $V$ such that $k \subset A, k \neq A, A W \subset W$ and

$$
r=\operatorname{rank} A=\text { G.C.D. }\{\text { ord } a \mid a \in A\} \text {. }
$$

We denote by $\mathcal{S}_{r}(\mu, \nu)$ the set of all Schur pairs of rank $r$, index $\mu$ and level $\nu$.

Remark 1.3. If $(A, W)$ is a Schur pair, then $W$ cannot be an arbitrary point of the Grassmannian. Let

$$
A_{W}=\{v \in V \mid v W \subset W\} .
$$

If $k \neq A_{W}$, then $\left(A_{W}, W\right)$ gives a Schur pair, which we call a maximal Schur pair. However, for a generic $W$, we always have $A_{W}=k$. In this case, $W$ does not have any interesting geometric informations. 
We define the set of finite rank points of the Grassmannian by

$$
G_{\text {fin }}(\mu, \nu)=\left\{W \in G(\mu, \nu) \mid A_{W} \neq k\right\}
$$

Then we have a canonical injection

$$
s: G_{\text {fin }}(\mu, \nu) \longrightarrow \bigcup_{r \in \mathbb{N}} \mathcal{S}_{r}(\mu, \nu)
$$

defined by $s(W)=\left(A_{W}, W\right)$, which we call the Schur map.

Definition 1.4. We define the category of Schur pairs $\mathcal{S}(\nu)$ for every $\nu \in \mathbb{Z}$ as follows:

(1) The set of objects is defined by

$$
O b(\mathcal{S}(\nu))=\bigcup_{\mu \in \mathbb{Z}} \bigcup_{r \in \mathbb{N}} \mathcal{S}_{r}(\mu, \nu)
$$

(2) The set of morphisms $\operatorname{Mor}\left(\left(A_{2}, W_{2}\right),\left(A_{1}, W_{1}\right)\right)$ consists of

$$
(\alpha, \iota):\left(A_{2}, W_{2}\right) \longrightarrow\left(A_{1}, W_{1}\right)
$$

where $\alpha: A_{2} \hookrightarrow A_{1}$ and $\iota: W_{2} \hookrightarrow W_{1}$ are inclusion maps.

\section{The category $\mathcal{Q}(\nu)$ of geometric data on algebraic curves.}

In this section we define categories of geometric data consisting of algebraic curves and torsion free sheaves on them, and show that every object of these categories gives rise to a Schur pair which is defined in the previous section.

Definition 2.1. Let $r>0$ be a positive integer, $\mu$ and $\nu$ be arbitrary integers. We call $(C, p, \mathcal{F}, \pi, \phi)$ a quintet of rank $r$, index $\mu$ and level $\nu$ if it consists of the following geometric data:

(1) $C$ is a reduced irreducible complete algebraic curve defined over a field $k$ of arbitrary characteristic;

(2) $p \in C$ is a smooth $k$-rational point;

(3) $\mathcal{F}$ is a torsion free sheaf of $\mathcal{O}_{C}$-modules on $C$ of rank $r$ satisfying

$$
\operatorname{dim}_{k} H^{0}(C, \mathcal{F})-\operatorname{dim}_{k} H^{1}(C, \mathcal{F})=\mu
$$

(4) $\pi: U_{0} \rightarrow U_{p}$ is a morphism of formal schemes, where $U_{0}$ is the formal completion of the affine line $\mathbb{A}_{k}^{1}=\mathbb{A}^{1}$ along the origin $0 \in \mathbb{A}^{1}$ and $U_{p}$ is the formal completion of the curve $C$ along $p$. Once for all, we choose a 
coordinate $z$ on $\mathbb{A}^{1}$ and fix it throughout this paper. Thus $U_{0}=\operatorname{Spec} k[[z]]$. The morphism $\pi$ induces a ring homomorphism

$$
\pi^{*}: \widehat{\mathcal{O}}_{C, p} \longrightarrow \widehat{\mathcal{O}}_{\mathbb{A}^{1}, 0}
$$

We require that $\pi^{*}$ is injective and the ideal of $\widehat{\mathcal{O}}_{\mathbb{A}^{1}, 0}$ generated by $\pi^{*}\left(m_{p}\right)$ coincides with the $r$-th power $\left(m_{0}\right)^{r}$ of the unique maximal ideal $m_{0}$ of $\widehat{\mathcal{O}}_{\mathbb{A}^{1}, 0}$, where $m_{p}$ is the unique maximal ideal of $\widehat{\mathcal{O}}_{C, p}$. We call such $\pi$ an $r$-sheeted covering of $U_{p}$ ramified at $p$.

(5) $\phi: \mathcal{F}_{U_{p}} \stackrel{\sim}{\longrightarrow} \pi_{*} \mathcal{O}_{U_{0}}(\nu)$ is an $\mathcal{O}_{U_{p}}$-module isomorphism between the formal completion $\mathcal{F}_{U_{p}}$ of $\mathcal{F}$ along $p \in C$ which is a free $\mathcal{O}_{U_{p}}$-module of rank $r$ and the direct image $\pi_{*} \mathcal{O}_{U_{0}}(\nu)$ of the twisted structure sheaf $\mathcal{O}_{U_{0}}(\nu)$ of the formal scheme $U_{0}$ via the morphism $\pi$. Since $\pi$ is an r-sheeted covering, $\pi_{*} \mathcal{O}_{U_{0}}(\nu)$ is a free $\mathcal{O}_{U_{p}}$-module of rank $r$.

Two quintets $(C, p, \mathcal{F}, \pi, \phi)$ and $(C, p, \mathcal{F}, \pi, c \phi)$ are identified if $c \in k^{\times}$. We also identify $\left(C, p, \mathcal{F}, \pi_{1}, \phi_{1}\right)$ with $\left(C, p, \mathcal{F}, \pi_{2}, \phi_{2}\right)$ if the following commutative diagram holds:

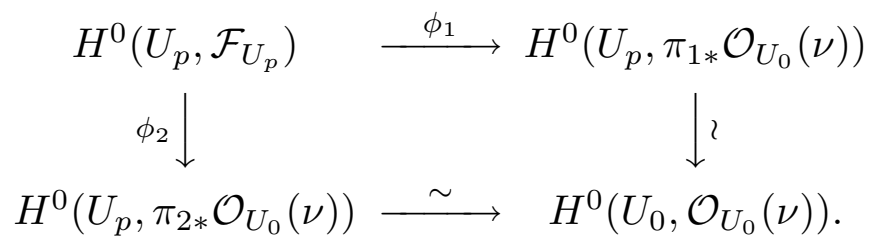

The set of all quintets of rank $r$, index $\mu$ and level $\nu$ is denoted by $\mathcal{Q}_{r}(\mu, \nu)$.

Remark 2.2.

(1) Let $\operatorname{Sing} C$ denote the singularity of $C$. Since $\operatorname{dim} C=1, \mathcal{F}$ is locally free on $C \backslash \operatorname{Sing} C$. In particular, if $C$ is a non-singular curve, then $\mathcal{F}$ is actually a vector bundle on $C$ of rank $r$ and degree $\mu+r(g-1)$, where $g$ is the genus of $C$. The last formula of degree follows from the Riemann-Roch theorem

$$
\operatorname{dim}_{k} H^{0}(C, \mathcal{F})-\operatorname{dim}_{k} H^{1}(C, \mathcal{F})=\operatorname{deg} \mathcal{F}-r(g-1) .
$$

(2) In the case of $k=\mathbb{C}$, we can use a local Stein neighborhood of $p$ in $C$ instead of the formal completion. Thus $U_{p}$ is a small open set of $C$ around $p$ and $U_{0}$ is a small open disk of the complex plane $\mathbb{C}$ centering at the origin. The morphism $\pi$ is a holomorphic covering of $U_{p}$ ramified at $p$. In this case we can replace the formal completion $\mathcal{F}_{U_{p}}$ by the restriction $\left.\mathcal{F}\right|_{U_{p}}$ and thus the map $\phi$ gives an isomorphism

$$
\phi:\left.\mathcal{F}\right|_{U_{p}} \stackrel{\sim}{\longrightarrow} \pi_{*} \mathcal{O}_{U_{0}}(\nu)
$$

as a trivial holomorphic vector bundle on $U_{p}$. In other words, $\pi$ is a local covering of the curve $C$ at the point $p$ and $\phi$ can be thought of as a local trivialization of the vector bundle $\mathcal{F}$ on $U_{p}$. 
(3) When $r=1, \pi$ gives an isomorphism $\pi: U_{0} \stackrel{\sim}{\longrightarrow} U_{p}$. Since we have chosen a coordinate $z$ on $U_{0}, \pi$ gives a local coordinate $y=\pi(z)$ on $U_{p}$. Thus our quintet $(C, p, \mathcal{F}, \pi, \phi)$ becomes $(C, p, \mathcal{F}, y, \phi)$ with a local parameter $y$ around $p$ and a local trivialization $\phi$ of $\mathcal{F}$ near the point $p$. This is the quintuple introduced by Segal-Wilson [SW].

Definition 2.3. We define a category $\mathcal{Q}(\nu)$ of quintets of level $\nu \in \mathbb{Z}$ as follows:

(1) The set of objects is defined by

$$
O b(\mathcal{Q}(\nu))=\bigcup_{\mu \in \mathbb{Z}} \bigcup_{r \in \mathbb{N}} \mathcal{Q}_{r}(\mu, \nu) .
$$

(2) A morphism

$$
(\beta, \psi):\left(C_{1}, p_{1}, \mathcal{F}_{1}, \pi_{1}, \phi_{1}\right) \longrightarrow\left(C_{2}, p_{2}, \mathcal{F}_{2}, \pi_{2}, \phi_{2}\right)
$$

consists of a morphism $\beta: C_{1} \rightarrow C_{2}$ of curves and a homomorphism $\psi$ : $\mathcal{F}_{2} \rightarrow \beta_{*} \mathcal{F}_{1}$ of sheaves on $C_{2}$ such that

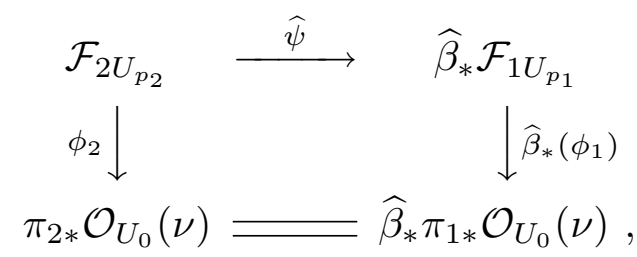

where $\widehat{\psi}$ is the homomorphism of sheaves on $U_{p_{2}}$ defined by $\psi$.

In the above definition of quintet, we introduced a fixed formal coordinate $z$ on the formal scheme $U_{0}$. We are going to define in the next section a contravariant functor $\chi_{(\nu)}$ from $\mathcal{Q}(\nu)$ to $\mathcal{S}(\nu)$ by using this coordinate. The formal parameter $z$ which is used in the definition of Grassmannians is nothing but the coordinate $z$ on $U_{0}$, and this is the key of the connection between the two completely different categories.

However, from geometric point of view, it is more desireble to have coordinate free objects. This is one of the motivations of introducing another category in the next definition. 
Definition 2.4. The thickened category $\overline{\mathcal{Q}}(\nu)$ of quintets of level $\nu \in \mathbb{Z}$ is defined as follows:

(1) The set of objects is exactly the same as before;

$$
O b(\overline{\mathcal{Q}}(\nu))=O b(\mathcal{Q}(\nu)) .
$$

(2) A morphism

$$
(\beta, \psi):\left(C_{1}, p_{1}, \mathcal{F}_{1}, \pi_{1}, \phi_{1}\right) \longrightarrow\left(C_{2}, p_{2}, \mathcal{F}_{2}, \pi_{2}, \phi_{2}\right)
$$

consists of a morphism $\beta: C_{1} \rightarrow C_{2}$ of curves and a homomorphism $\psi$ : $\mathcal{F}_{2} \rightarrow \beta_{*} \mathcal{F}_{1}$ of sheaves on $C_{2}$ satisfying the following four conditions:

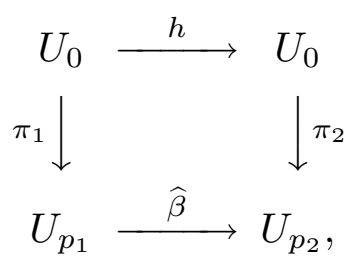

where $\widehat{\beta}$ is the morphism of formal schemes determined by $\beta$. There is an $\mathcal{O}_{U_{0}}$-module isomorphism $\xi: \mathcal{O}_{U_{0}}(\nu) \stackrel{\sim}{\rightarrow} h_{*} \mathcal{O}_{U_{0}}(\nu)$ such that

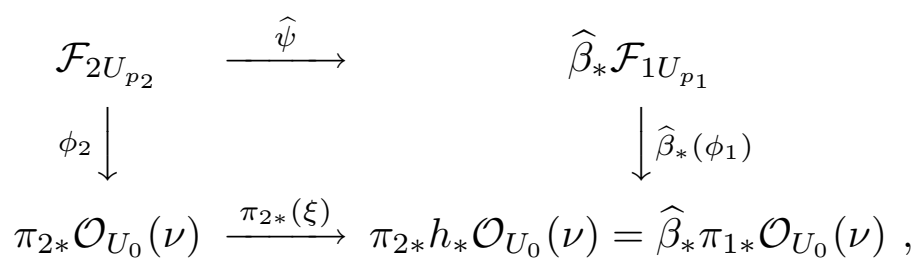

where $\widehat{\psi}$ is the homomorphism of sheaves on $U_{p_{2}}$ defined by $\psi$.

The new category $\overline{\mathcal{Q}}(\nu)$ has the same objects of $\mathcal{Q}(\nu)$ but has much more morphisms than that. It is very interesting to know, as we are going to see in Section 5, that this thickened category has a direct connection with the commutative algebras of ordinary differential operators. 
Definition 2.5. Let $U \subset C$ be an affine open subset of $C$ containing $p \in C$ and let $U_{p}$ be the formal completion of $C$ along $p$. For every coherent sheaf $\mathcal{L}$ on $U$, we define

$$
H^{0}(U \backslash\{p\}, \mathcal{L})=\varliminf_{n} H^{0}\left(U, \mathcal{L} \otimes \mathcal{O}_{U}(n)\right)
$$

and

$$
H^{0}\left(U_{p} \backslash\{p\}, \mathcal{L}_{U_{p}}\right)=\varliminf_{n} H^{0}\left(U_{p}, \mathcal{L}_{U_{p}} \otimes \mathcal{O}_{U_{p}}(n)\right)
$$

These are the set of regular sections of $\mathcal{L}$ defined on $U \backslash\{p\}$ or $U_{p} \backslash\{p\}$ which have poles of finite order at $p \in C$. Since $p$ is a smooth $k$-rational point of $C$, there is a canonical inclusion $\mathcal{L}_{p} \subset \mathcal{L}_{U_{p}}$. Therefore, we can view the cohomology groups such as $H^{0}(U \backslash\{p\}, \mathcal{L}), H^{0}\left(U_{p}, \mathcal{L}_{U_{p}}\right)$ and $H^{0}(U, \mathcal{L})$ as subgroups of $H^{0}\left(U_{p} \backslash\{p\}, \mathcal{L}_{U_{p}}\right)$. Now we have

\section{Proposition 2.6.}

(1) Let $\mathcal{L}$ be a torsion free sheaf on a Zariski open set $U \subset C$ containing $p$. Then

$$
H^{0}(U \backslash\{p\}, \mathcal{L}) \cap H^{0}\left(U_{p}, \mathcal{L}_{U_{p}}\right)=H^{0}(U, \mathcal{L}) .
$$

(2) There is a Zariski open set $U \subset C$ containing $p \in C$ so that we have a canonical isomorphism

$$
H^{0}\left(U \backslash\{p\}, \mathcal{O}_{U}\right) / H^{0}\left(U, \mathcal{O}_{U}\right) \stackrel{\sim}{\longrightarrow} H^{0}\left(U_{p} \backslash\{p\}, \mathcal{O}_{U_{p}}\right) / H^{0}\left(U_{p}, \mathcal{O}_{U_{p}}\right) .
$$

(3) Let $\mathcal{F}$ be a torsion free sheaf on $C$. Then there exists a Zariski open set $U \subset C$ containing $p \in C$ such that

$$
H^{0}(U \backslash\{p\}, \mathcal{F}) / H^{0}(U, \mathcal{F}) \stackrel{\sim}{\longrightarrow} H^{0}\left(U_{p} \backslash\{p\}, \mathcal{F}_{U_{p}}\right) / H^{0}\left(U_{p}, \mathcal{F}_{U_{p}}\right)
$$

gives a natural isomorphism.

Proof.

(1) Every regular section of $\mathcal{L}$ defined on $U \backslash\{p\}$ which is also regular at $p$ is regular everywhere on $U$.

(2) Since $p \in C$ is a smooth $k$-rational point, there is a rational function $y$ on $C$ which has a simple zero at $p$. Let $U$ be a Zariski open set of $C$ such that $y$ is regular everywhere on $U$ and $y^{-1}$ is regular on $U$ except at $p$. Since the equation $y=0$ on $U$ defines the point $p \in U \subset C, y$ is the (topological) generator of $\widehat{\mathcal{O}}_{C, p}=\mathcal{O}_{U_{p}}$, i.e. $H^{0}\left(U_{p}, \mathcal{O}_{U_{p}}\right)=k[[y]]$. Therefore,

$$
H^{0}\left(U_{p} \backslash\{p\}, \mathcal{O}_{U_{p}}\right)=\varliminf_{n} H_{12}^{0}\left(U_{p}, \mathcal{O}_{U_{p}}(n)\right)=k((y)) .
$$


On the other hand, since $y^{\nu} \in H^{0}\left(U \backslash\{p\}, \mathcal{O}_{U}\right)$ for every $\nu \in \mathbb{Z}$, we have

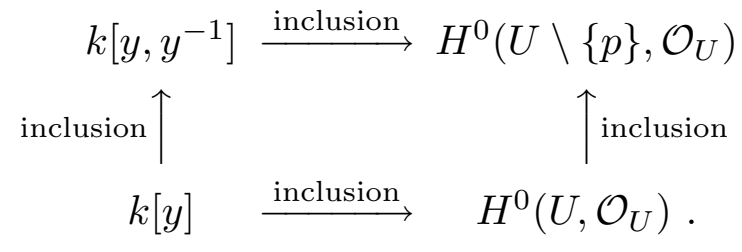

But since $k\left[y, y^{-1}\right] \cap H^{0}\left(U, \mathcal{O}_{U}\right)=k[y]$, there is a natural inclusion

$$
k\left[y, y^{-1}\right] / k[y] \hookrightarrow H^{0}\left(U \backslash\{p\}, \mathcal{O}_{U}\right) / H^{0}\left(U, \mathcal{O}_{U}\right) .
$$

Similarly, since we have

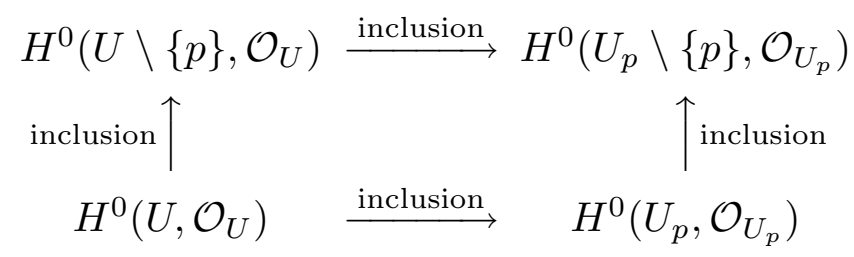

and

$$
H^{0}\left(U \backslash\{p\}, \mathcal{O}_{U}\right) \cap H^{0}\left(U_{p}, \mathcal{O}_{U_{p}}\right)=H^{0}\left(U, \mathcal{O}_{U}\right),
$$

there is a natural inclusion

$$
H^{0}\left(U \backslash\{p\}, \mathcal{O}_{U}\right) / H^{0}\left(U, \mathcal{O}_{U}\right) \hookrightarrow H^{0}\left(U_{p} \backslash\{p\}, \mathcal{O}_{U_{p}}\right) / H^{0}\left(U_{p}, \mathcal{O}_{U_{p}}\right) .
$$

By combining these, we obtain

$$
\begin{aligned}
k\left[y, y^{-1}\right] / k[y] & \hookrightarrow H^{0}\left(U \backslash\{p\}, \mathcal{O}_{U}\right) / H^{0}\left(U, \mathcal{O}_{U}\right) \\
& \hookrightarrow H^{0}\left(U_{p} \backslash\{p\}, \mathcal{O}_{U_{p}}\right) / H^{0}\left(U_{p}, \mathcal{O}_{U_{p}}\right) \\
& =k((y)) / k[[y]] .
\end{aligned}
$$

But note that $k\left[y, y^{-1}\right] / k[y] \simeq k((y)) / k[[y]]$. Therefore, every injective homomorphism in the above is indeed an isomorphism.

(3) Let $U$ be an affine open subset of $C$ on which $\mathcal{F}$ is locally free. Since $p$ is a smooth point of $C, U$ contains $p$. If the rank of $\mathcal{F}$ is $r$, then we have an isomorphism $\eta:\left.\mathcal{F}\right|_{U} \stackrel{\sim}{\longrightarrow}\left(\mathcal{O}_{U}\right)^{r}$. Thus

$$
H^{0}(U \backslash\{p\}, \mathcal{F}) / H^{0}(U, \mathcal{F}) \simeq\left(H^{0}\left(U \backslash\{p\}, \mathcal{O}_{U}\right) / H^{0}\left(U, \mathcal{O}_{U}\right)\right)^{r} .
$$

We can take $U$ even smaller so that the above (2) holds. Then

$$
\begin{aligned}
H^{0}(U \backslash\{p\}, \mathcal{F}) / H^{0}(U, \mathcal{F}) & \simeq\left(H^{0}\left(U \backslash\{p\}, \mathcal{O}_{U}\right) / H^{0}\left(U, \mathcal{O}_{U}\right)\right)^{r} \\
& =\left(H^{0}\left(U_{p} \backslash\{p\}, \mathcal{O}_{U_{p}}\right) / H^{0}\left(U_{p}, \mathcal{O}_{U_{p}}\right)\right)^{r} \\
& \simeq H^{0}\left(U_{p} \backslash\{p\}, \mathcal{F}_{U_{p}}\right) / H^{0}\left(U_{p}, \mathcal{F}_{U_{p}}\right),
\end{aligned}
$$

because the formal completion of $\eta$ gives a trivialization $\widehat{\eta}: \mathcal{F}_{U_{p}} \stackrel{\sim}{\longrightarrow}\left(\mathcal{O}_{U_{p}}\right)^{r}$. The resulting isomorphism between the quotient of cohomologies does not depend on the choice of the trivialization $\eta$. 
This completes the proof of the proposition.

In the above proof, we have established that

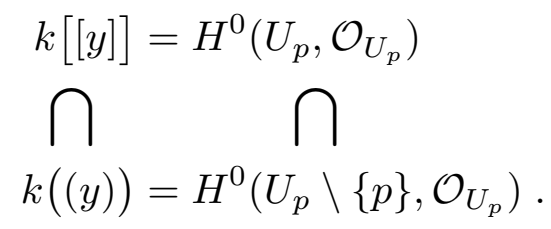

Note that we have chosen a fixed coordinate $z$ on $U_{0}$. Therefore, the ring homomorphism $\pi^{*}$ associated to the morphism $\pi: U_{0} \rightarrow U_{p}$ gives an injection

$$
k[[y]]=H^{0}\left(U_{p}, \mathcal{O}_{U_{p}}\right) \stackrel{\pi^{*}}{\longrightarrow} H^{0}\left(U_{0}, \mathcal{O}_{U_{0}}\right)=k[[z]]
$$

This injective ring homomorphism $\pi^{*}: k[[y]] \hookrightarrow k[[z]]$ extends to a unique injective field homomorphism $\pi^{*}: k((y)) \hookrightarrow k((z))$ over $k$ which we denote by the same notation $\pi^{*}$. Thus we have obtained an extended diagram

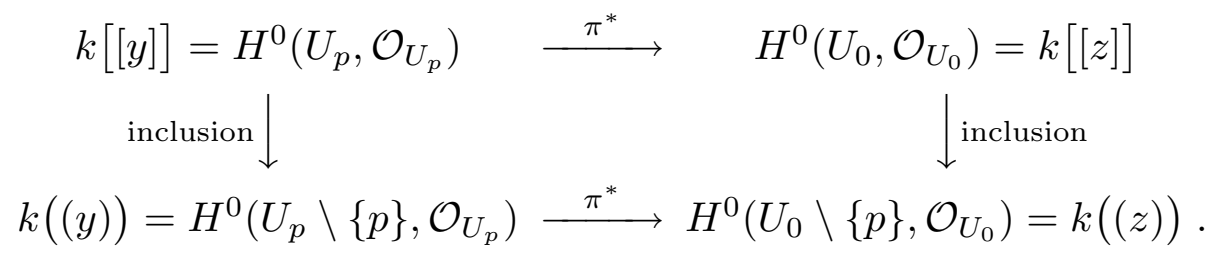

In particular, $\pi^{*}\left(H^{0}\left(C \backslash\{p\}, \mathcal{O}_{C}\right)\right)$ becomes a well-defined subring of $k((z))$. Similarly, since

$$
H^{0}\left(U_{p}, \pi_{*} \mathcal{O}_{U_{p}}(\nu)\right) \simeq H^{0}\left(U_{0}, \mathcal{O}_{U_{0}}(\nu)\right)=V^{(\nu)},
$$

$\phi: \mathcal{F}_{U_{p}} \stackrel{\sim}{\rightarrow} \pi_{*} \mathcal{O}_{U_{0}}(\nu)$ induces an isomorphism $\phi: H^{0}\left(U_{p}, \mathcal{F}_{U_{p}}\right) \stackrel{\sim}{\rightarrow} V^{(\nu)}$. By using the fact that $\pi_{*} \mathcal{O}_{U_{0}}(\nu)$ is a free $\mathcal{O}_{U_{p}}$-module, we have

$$
\begin{aligned}
H^{0}\left(U_{p}^{*}, \pi_{*} \mathcal{O}_{U_{p}}(\nu)\right) & =\underset{n}{\lim _{n}} H^{0}\left(U_{p}, \pi_{*} \mathcal{O}_{U_{p}}(\nu) \underset{\mathcal{O}_{U_{p}}}{\otimes} \mathcal{O}_{U_{p}}(n)\right) \\
& \simeq H^{0}\left(U_{p}, \pi_{*} \mathcal{O}_{U_{p}}(\nu)\right)_{H^{0}\left(U_{p}, \mathcal{O}_{U_{p}}\right)}^{\otimes} H^{0}\left(U_{p}^{*}, \mathcal{O}_{U_{p}}\right) \\
& \simeq V^{(\nu)} \quad \underset{k[[y]]}{\otimes} k((y)) \\
& =k((z)),
\end{aligned}
$$

where $U_{p}^{*}=U_{p} \backslash\{p\}$. Therefore, we obtain extended isomorphisms

$$
H^{0}\left(U_{p} \backslash\{p\}, \mathcal{F}_{U_{p}}\right) \stackrel{\sim}{\longrightarrow} H^{0}\left(U_{p} \backslash\{p\}, \pi_{*} \mathcal{O}_{U_{p}}(\nu)\right) \stackrel{\sim}{\longrightarrow} k((z))
$$


which we denote by the same notation $\phi$. Thus we have

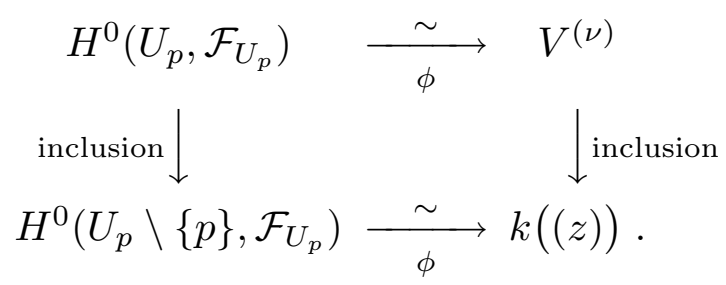

In particular, $\phi\left(H^{0}(C \backslash\{p\}, \mathcal{F})\right)$ becomes a well-defined subset of $k((z))$.

Theorem 2.7. For every quintet $(C, p, \mathcal{F}, \pi, \phi)$ of level $\nu$, let us define

$$
W=\phi\left(H^{0}(C \backslash\{p\}, \mathcal{F})\right) \subset k((z))
$$

Then we have

$$
\operatorname{Ker} \gamma(\nu)_{W} \simeq H^{0}(C, \mathcal{F}) \text { and } \operatorname{Coker} \gamma(\nu)_{W} \simeq H^{1}(C, \mathcal{F})
$$

where $\gamma(\nu)_{W}$ is the natural map of (1.3).

Proof. Let $U \subset C$ be an affine open subset of $C$ containing $p$. Since $C \backslash\{p\}$ is an affine subset of $C$, we can compute the cohomology by using the affine covering $C=(C \backslash\{p\}) \cup U$. Because of Proposition 2.6 (1), we have

$$
\begin{aligned}
H^{0}(C, \mathcal{F}) & \simeq H^{0}(C \backslash\{p\}, \mathcal{F}) \cap H^{0}\left(U_{p}, \mathcal{F}_{U_{p}}\right) \\
& \simeq \phi\left(H^{0}(C \backslash\{p\}, \mathcal{F})\right) \cap \phi\left(H^{0}\left(U_{p}, \mathcal{F}_{U_{p}}\right)\right) \\
& =W \cap V^{(\nu)} \\
& =\operatorname{Ker} \gamma(\nu)_{W}
\end{aligned}
$$

Next, let $U \subset C$ be an affine open subset of $C$ containing $p$ such that $\mathcal{F}$ is locally free on $U$. Then, by Proposition 2.6 (3) we have

$$
\begin{aligned}
H^{1}(C, \mathcal{F}) & \simeq H^{0}(U \backslash\{p\}, \mathcal{F}) /\left(H^{0}(C \backslash\{p\}, \mathcal{F})+H^{0}(U, \mathcal{F})\right) \\
& \simeq H^{0}\left(U_{p} \backslash\{p\}, \mathcal{F}_{U_{p}}\right) /\left(H^{0}(C \backslash\{p\}, \mathcal{F})+H^{0}\left(U_{p}, \mathcal{F}_{U_{p}}\right)\right) \\
& \simeq \phi\left(H^{0}\left(U_{p} \backslash\{p\}, \mathcal{F}_{U_{p}}\right)\right) / \phi\left(H^{0}(C \backslash\{p\}, \mathcal{F})+H^{0}\left(U_{p}, \mathcal{F}_{U_{p}}\right)\right) \\
& =k((z)) /\left(W+V^{(\nu)}\right) \\
& \simeq \operatorname{Coker} \gamma(\nu)_{W} .
\end{aligned}
$$

This completes the proof. 
Corollary 2.8. Let $q=(C, p, \mathcal{F}, \pi, \phi) \in \mathcal{Q}_{r}(\mu, \nu)$ be a quintet of rank $r$, index $\mu$ and level $\nu$. Define a Schur pair by $\chi_{r, \mu, \nu}(q)=(A, W)$, where $A=\pi^{*}\left(H^{0}(C \backslash\right.$ $\left.\left.\{p\}, \mathcal{O}_{C}\right)\right)$ and $W=\phi\left(H^{0}(C \backslash\{p\}, \mathcal{F})\right)$. Then $(A, W) \in \mathcal{S}_{r}(\mu, \nu)$ and we have a well-defined map

$$
\chi_{r, \mu, \nu}: \mathcal{Q}_{r}(\mu, \nu) \longrightarrow \mathcal{S}_{r}(\mu, \nu)
$$

for every $r>0, \mu, \nu \in \mathbb{Z}$.

Proof. Since $H^{0}\left(C \backslash\{p\}, \mathcal{O}_{C}\right) \neq k$, we know that $A \neq k$. Note that $\mathcal{F}$ is an $\mathcal{O}_{C^{-}}$ module and that $\phi: \mathcal{F}_{U_{p}} \stackrel{\sim}{\longrightarrow} \pi_{*} \mathcal{O}_{U_{0}}(\nu)$ is an $\mathcal{O}_{U_{p}}$-module isomorphism. Therefore, $W$ is an $A$-module, i.e. $A W \subset W$. As in the proof of Proposition 2.6 (2), let $y$ be a rational function on $C$ which has a simple zero at $p$. Then we have

$$
H^{0}\left(C \backslash\{p\}, \mathcal{O}_{C}\right) \subset H^{0}\left(U_{p} \backslash\{p\}, \mathcal{O}_{U_{p}}\right)=k((y)) \underset{\pi^{*}}{\hookrightarrow} k((z)) .
$$

Since $\pi: U_{0} \rightarrow U_{p}$ is an $r$-sheeted covering ramified at $p$, the image $\pi^{*}(y)$ of $y$ is an element of $k[[z]]$ of order $-r$. Therefore, rank $A=r$.

On the other hand, since

$$
\operatorname{Index} \gamma(\nu)_{W}=\operatorname{dim}_{k} H^{0}(C, \mathcal{F})-\operatorname{dim}_{k} H^{1}(C, \mathcal{F})=\mu,
$$

we have $W \in G(\mu, \nu)$. Therefore,

$$
\chi_{r, \mu, \nu}(q)=(A, W) \in \mathcal{S}_{r}(\mu, \nu) .
$$

This completes the proof.

\section{Categorical equivalence of $\mathcal{S}(\nu)$ and $\mathcal{Q}(\nu)$.}

In the previous section we defined a map

$$
\chi_{r, \mu, \nu}: \mathcal{Q}_{r}(\mu, \nu) \longrightarrow \mathcal{S}_{r}(\mu, \nu)
$$

from the set of quintets into the set of Schur pairs for every rank $r$, index $\mu$ and level $\nu$. In this section, we are going to extend this map to a contravariant functor $\chi_{(\nu)}: \mathcal{Q}(\nu) \rightarrow \mathcal{S}(\nu)$ which makes these categories (anti-) equivalent. In order to establish the categorical equivalence, we have to show bijectivity of $\chi_{r, \mu, \nu}$. This will be done by giving an explicit construction of a quintet starting from a Schur pair. In other words, we will define the inverse map of $\chi_{r, \mu, \nu}$.

The main idea of constructing a quintet out of a Schur pair $(A, W)$ is the following. First of all, we introduce a natural filtration in $A$ by using the filtration (1.1) of $V$. Then we define the graded algebra gr $A$ corresponding to $A$. Now the curve is obtained by $C=\operatorname{Proj}(\operatorname{gr} A)$ and we will show that it is a one-point completion of an affine curve $\operatorname{Spec} A$. Thus $\operatorname{Proj}(\operatorname{gr} A)=\operatorname{Spec} A \cup\{p\}$. The $A$-module structure 
on $W$ defines a torsion free sheaf on the affine curve Spec $A$ and the level structure of $W$ determines the extension of this sheaf to a sheaf on $C$. The other data $\pi$ and $\phi$ are constructed by identifying the formal variable $z$ appearing in the definition of Grassmannians with a local coordinate of $C$ near the point $p$.

Now let us start with a Schur pair $(A, W) \in \mathcal{S}_{r}(\mu, \nu)$ of rank $r>0$, index $\mu$ and level $\nu$. We define the natural filtrations of $A$ and $W$ by $A^{(n)}=A \cap V^{(n)}$ and $W^{(n)}=W \cap V^{(n)}$. Since $\operatorname{Ker} \gamma(\nu)_{W}=W \cap V^{(\nu)}$ is of finite dimension, there is a well-defined number

$$
M=\min \{\operatorname{ord} w \mid w \in W\}
$$

such that $W^{(M)} \neq\{0\}$ and $W^{(M-1)}=\{0\}$. Since $\operatorname{dim}_{k} V^{(n)} / V^{(n-1)}=1$ for every $n \in \mathbb{Z}$, we have

$$
1 \leq \operatorname{dim}_{k} W^{(M)}=\operatorname{dim}_{k} W^{(M)} / W^{(M-1)} \leq 1
$$

hence $\operatorname{dim}_{k} W^{(M)}=1$. Note that the multiplication in $V$ satisfies $V^{(m)} V^{(n)} \subset$ $V^{(m+n)}$. So we have $A^{(-1)} \cdot W^{(M)} \subset W^{(M-1)}=0$ and which means that $A^{(-1)}=0$. Moreover, since $A^{(0)} \cdot W^{(M)} \subset W^{(M)}, A^{(0)}$ must preserve the one-dimensional subspace $W^{(M)}$ of $V$. Therefore, we can conclude that

$$
A^{(0)}=A \cap V^{(0)}=k \text {. }
$$

Let

$$
K=\left\{a^{-1} b \mid a, b \in A \text { and } a \neq 0\right\} \subset V
$$

be the field of quotients of $A$. Every element of $K$ has an order which is divisible by $r=\operatorname{rank} A$, because the order of elements of $A$ is a multiple of $r$. Namely, we have

$$
\operatorname{dim}_{k} A^{(n r)} / A^{(n r-r)} \leq 1
$$

and

$$
\operatorname{dim}_{k} K^{(n r)} / K^{(n r-r)} \leq 1
$$

for every $n \in \mathbb{Z}$, where we define $K^{(n)}=K \cap V^{(n)}$.

Proposition 3.1. Let $(A, W) \in \mathcal{S}_{r}(\mu, \nu)$ be a Schur pair. Then there is a monic element $y \in K^{(-r)}$ of order $-r$ such that $A \subset K \subset k((y)) \subset k((z))$ and

$$
A \cap k[[y]]=k
$$

Proof. Let $a$ be an arbitrary monic element of $A$ of positive order, i.e. the leading coefficient of $a$ is 1 and ord $a>0$. Since

$$
r=\underset{G . C . D .}{\operatorname{sord} v \mid v \in A\},},
$$


there is another monic elements $b$ in $A$ such that

$$
r=i(\operatorname{ord} a)-j(\operatorname{ord} b)
$$

for some positive integers $i$ and $j$. So define

$$
y=a^{-i} b^{j} \in K,
$$

which is a monic element of $K$ of order $-r$ because of (3.3). Thus $y \in K \cap V^{(-r)} \subset$ $K \cap k[[z]]$. Since $k(y) \subset K$, we have

$$
\operatorname{dim}_{k} K^{(n r)} / K^{(n r-r)}=1
$$

for every $n \in \mathbb{Z}$, because $y^{-n}$ gives a monic element of $K$ of order $n r$.

Let $v \in K^{(n r)}$ be an arbitrary element. Then we can choose a constant $c_{0} \in k$ so that $v-c_{0} y^{-n} \in K^{(n r-r)}$. Since $y^{-n+1}$ is a monic element of order $n r-r$, there is another constant $c_{1}$ such that $v-c_{0} y^{-n}-c_{1} y^{-n+1} \in K^{(n r-2 r)}$. If we continue this procedure, then we have a sequence of constants $c_{0}, c_{1}, c_{2}, \cdots$ such that

$$
v-\sum_{\ell=0}^{\infty} c_{\ell} y^{-n+\ell} \in \bigcap_{m \in \mathbb{Z}} V^{(m)}=\{0\} .
$$

Therefore, $v=\sum_{\ell=0}^{\infty} c_{\ell} y^{-n+\ell}$, and which implies that $k(y) \subset K \subset k((y))$. Note that $k((y)) \cap V^{(0)}=k[[y]]$ and $A \subset K \subset k((y))$. Thus

$$
\begin{aligned}
A \cap k[[y]] & =A \cap k((y)) \cap V^{(0)} \\
& =A \cap V^{(0)} \\
& =k
\end{aligned}
$$

by (3.1). This completes the proof.

In order to construct a curve out of the algebra $A$, we have to show that $A$ has dimension one over $k$.

Proposition 3.2. Let $N_{A}=\{\operatorname{ord} v \mid v \in A\} \subset \mathbb{N} \cup\{0\}$ be the set of the order of elements of $A$. Then there exists a finite subset $F_{A}$ of $\mathbb{N}$ such that

$$
N_{A}=r\left(\mathbb{N} \backslash F_{A}\right) \cup\{0\} .
$$

Moreover, the algebra $A$ has dimension one over $k$.

Proof. Let $a$ and $b$ be the same elements used in the proof of Proposition 3.1, and let $\alpha=\operatorname{ord} a, \beta=\operatorname{ord} b$. Recall that $i \alpha-j \beta=r$ by (3.3). As we have observed 
already, $A^{(-1)}=0$. Therefore, $N_{A} \subset r \mathbb{N} \cup\{0\}$. In order to establish (3.4), it suffices to show that $N_{A}$ contains arbitrary large multiple of $r$. So let $\alpha=r \alpha^{\prime}$ and $\beta=r \beta^{\prime}$. We claim that

$$
r n \in N_{A} \quad \text { for every } n \geq j \alpha^{\prime} \beta^{\prime} .
$$

Indeed, let $n$ be an arbitrary integer greater than $j \alpha^{\prime} \beta^{\prime}$. Applying the Euclid division algorithm to $n-j \alpha^{\prime} \beta^{\prime}$, we find unique integers $m \geq 0$ and $0 \leq \ell<\alpha^{\prime}$ such that

$$
n=j \alpha^{\prime} \beta^{\prime}+m \alpha^{\prime}+\ell .
$$

Therefore,

$$
\begin{aligned}
r n & =r j \alpha^{\prime} \beta^{\prime}+r m \alpha^{\prime}+r \ell \\
& =j \alpha^{\prime} \beta+m \alpha+\ell(i \alpha-j \beta) \\
& =(m+i \ell) \alpha+\left(\alpha^{\prime}-\ell\right) j \beta \\
& =\operatorname{ord}\left(a^{m+i \ell} \cdot b^{\left(\alpha^{\prime}-\ell\right) j}\right) \\
& \in N_{A} .
\end{aligned}
$$

This establishes (3.5). Now define $F_{A}=\left\{n \in \mathbb{N} \mid r n \notin N_{A}\right\}$. Then $F_{A}$ is a finite set, since its cardinality $\# F_{A}$ is clearly smaller than $j \alpha^{\prime} \beta^{\prime}$. This establises (3.4).

The above argument shows that

$$
\operatorname{dim}_{k} A^{(r n)} / A^{(r n-r)}=1 \text { for all } n \geq j \alpha^{\prime} \beta^{\prime} .
$$

But since we used only $a$ and $b$, we also have

$$
\operatorname{dim}_{k} k[a, b]^{(n r)} / k[a, b]^{(n r-r)}=1 \text { for all } n \geq j \alpha^{\prime} \beta^{\prime},
$$

where we define $k[a, b]^{(n)}=k[a, b] \cap V^{(n)}$ and $k[a, b]$ denotes the $k$-subalgebra of $V$ generated by $1, a$ and $b$. Therefore, we have

$$
\operatorname{dim}_{k} A / k[a, b] \leq \# F_{A}<j \alpha^{\prime} \beta^{\prime} .
$$

This means that the dimension of $A$ over $k$ is at most two.

Now we have to show that $a$ and $b$ satisfy a non-trivial polynomial relation. So let us assume that $b \notin k[a]$. Let $\left\{u_{1}, \cdots, u_{q}\right\}$ be a $k$-linear basis for $k[a, b]^{\left(r j \alpha^{\prime} \beta^{\prime}-r\right)}$ and let

$$
v_{n}=a^{m+i \ell} \cdot b^{\left(\alpha^{\prime}-\ell\right) j} \quad \text { for } n \geq j \alpha^{\prime} \beta^{\prime},
$$

where $m$ and $\ell$ are the unique integers of (3.6). Then $\left\{u_{1}, u_{2}, \cdots, u_{q}\right\} \cup\left\{v_{n}\right\}_{n \geq j \alpha^{\prime} \beta^{\prime}}$ gives a $k$-linear basis for $k[a, b]$. Since none of the $v_{n}$ 's are powers of $a$ and $u_{m}$ 's can contain only finitely many powers of $a$, a very high power $a^{N}$ for some large 
number $N$ must be represented by a non-trivial linear combination of the basis vectors $u_{1}, u_{2}, \cdots, u_{q}$ and $v_{n}$ 's. But this is nothing but a non-trivial polynomial relation which $a$ and $b$ satisfy. Therefore, $k[a, b]$ has dimension one over $k$. Hence so does $A$. This completes the proof.

In order to define a complete algebraic curve over $k$, we need a graded algebra defined by

$$
\operatorname{gr} A=\bigoplus_{n=0}^{\infty} A^{(n)}
$$

Theorem 3.3. Let gr $A$ be the graded algebra defined in the above. Then $C=$ $\operatorname{Proj}(\operatorname{gr} A)$ is a reduced irreducible complete algebraic curve over $k$ of genus $\# F_{A}$, where $F_{A}$ is the finite set of Proposition 3.2. Moreover, there is a smooth $k$-rational point $p$ on $C$ such that

$$
C=\operatorname{Spec} A \cup\{p\}
$$

Proof. Let $a \in A$ be the element used in the proof of Proposition 3.2. We denote by gr $A\left[a^{-1}\right]$ the graded algebra generated by $a^{-1}$ over gr $A$ and by (gr $\left.A\left[a^{-1}\right]\right)_{0}$ its homogeneous degree zero part. We have a natural identification

$$
\left(\operatorname{gr} A\left[a^{-1}\right]\right)_{0}=\left\{a^{-\ell} v \mid \ell \geq 0, v \in A \text { and } \operatorname{ord}\left(a^{-\ell} v\right) \leq 0\right\} .
$$

Since the monic element $y=a^{-i} b^{j}$ is of order $-r$, it is an element of $\left(\operatorname{gr} A\left[a^{-1}\right]\right)_{0}$. Therefore, $k[y] \subset\left(\operatorname{gr} A\left[a^{-1}\right]\right)_{0}$. On the other hand, since

$$
\left(\operatorname{gr} A\left[a^{-1}\right]\right)_{0} \subset K^{(0)} \subset\left(k((y)) \cap V^{(0)}\right)=k[[y]]
$$

because of the above identification, we have

$$
k[y] \subset\left(\operatorname{gr} A\left[a^{-1}\right]\right)_{0} \subset k[[y]] .
$$

Therefore, the $(y)$-adic completion of $\left(\operatorname{gr} A\left[a^{-1}\right]\right)_{0}$ is equal to $k[[y]]$. So we define

$$
p=k[[y]] y \cap\left(\operatorname{gr} A\left[a^{-1}\right]\right)_{0},
$$

which is the maximal ideal of $\left(\operatorname{gr} A\left[a^{-1}\right]\right)_{0}$ generated by $y$. Let

$$
D_{+}(a)=\operatorname{Spec}\left(\operatorname{gr} A\left[a^{-1}\right]\right)_{0}
$$

This is an affine open subscheme of the projective scheme $\operatorname{Proj}(\operatorname{gr} A)=C$. Since $y$ is a rational function on $D_{+}(a)$ vanishing at $p \in D_{+}(a), p$ is a smooth $k$-rational point of $D_{+}(a) \subset C$. 
The relation $A \cap k[[y]]=k$ of Proposition 3.1 means that every rational function on $C$ which is regular on $\operatorname{Spec} A$ as well as at the point $p$ is a constant. Therefore, Spec $A \cup\{p\}$ has no missing point in the complete curve $C$ and which implies $C=\operatorname{Spec} A \cup\{p\}$. Note that $A$ is contained in the field $V=k((z))$. Therefore, Spec $A$ is reduced and irreducible, and hence so is $C$, because the attached point $p$ is a smooth point of $C$.

We have thus established that $C$ is a reduced irreducible complete curve and $p$ is a smooth $k$-rational point on it. Now we can apply Proposition 2.6 (1) and (2) in our situation. Let $U \subset C$ be an affine open subset of $C$ containing $p$ such that both of $y$ and $y^{-1}$ are regular on $U \backslash\{p\}$. Then we can compute the cohomology of $C$ by the affine covering $C=\operatorname{Spec} A \cup U$ so that we have

$$
\begin{aligned}
H^{1}\left(C, \mathcal{O}_{C}\right) & \simeq H^{0}\left(U \backslash\{p\}, \mathcal{O}_{C}\right) /\left(H^{0}\left(C \backslash\{p\}, \mathcal{O}_{C}\right)+H^{0}\left(U, \mathcal{O}_{U}\right)\right) \\
& \simeq H^{0}\left(U_{p} \backslash\{p\}, \mathcal{O}_{U_{p}}\right) /\left(H^{0}\left(C \backslash\{p\}, \mathcal{O}_{C}\right)+H^{0}\left(U_{p}, \mathcal{O}_{U_{p}}\right)\right) \\
& =k((y)) /(A+k[[y]]),
\end{aligned}
$$

where $U_{p}=\operatorname{Spec} k[[y]]$. It is clear from (3.4) that

$$
\operatorname{dim}_{k} H^{1}\left(C, \mathcal{O}_{C}\right)=\# F_{A} .
$$

Thus the (arithmetic) genus of the curve $C$ is the cardinality $\# F_{A}$ of $F_{A}$. This completes the proof.

In the above proof, we obtained that

$$
k[y] \subset\left(\operatorname{gr} A\left[a^{-1}\right]\right)_{0} \subset k[[y]] .
$$

Therefore, $U_{p}$ is the formal completion of $\operatorname{Spec}\left(\operatorname{gr} A\left[a^{-1}\right]\right)_{0}=D_{+}(a)$ along the point $p$. Since $D_{+}(a)$ is a Zariski open subset of $C, U_{p}$ is also a completion of $C$ along $p \in C$.

Recall that $y=a^{-i} b^{j}$ is a monic element of $k[[z]]$ of order $-r$. Therefore, there is a natural inclusion map $\pi^{*}: k[[y]] \hookrightarrow k[[z]]$ which defines a morphism

$$
\pi: U_{0} \longrightarrow U_{p}
$$

between the formal schemes $U_{0}=\operatorname{Spec} k[[z]]$ and $U_{p}=\operatorname{Spec} k[[y]]$. Since $U_{0}$ can be thought of as the formal completion of the affine $z$-line $\mathbb{A}_{k}^{1}$ along the origin, the morphism $\pi$ satisfies the condition in item (4) of Definition 2.1. Let us now construct a sheaf $\mathcal{F}$ on $C$ and its local trivialization $\phi$.

Theorem 3.4. Let $(A, W) \in \mathcal{S}_{r}(\mu, \nu)$ be a Schur pair of rank $r$, index $\mu$ and level $\nu$, and let $C=\operatorname{Proj}(\operatorname{gr} A)$ be the curve defined in Theorem 3.3. Then there exist a torsion free sheaf $\mathcal{F}$ of $\mathcal{O}_{C}$-modules of rank $r$ on $C$ satisfying

$$
H^{0}(C, \mathcal{F}) \simeq \operatorname{Ker} \gamma(\nu)_{W} \text { and } H^{1}(C, \mathcal{F}) \simeq \operatorname{Coker} \gamma(\nu)_{W},
$$


and an $\mathcal{O}_{U_{p}}$-module isomorphism

$$
\phi: \mathcal{F}_{U_{p}} \stackrel{\sim}{\longrightarrow} \pi_{*} \mathcal{O}_{U_{0}}(\nu)
$$

where $U_{p}$ is the formal completion of $C$ along $p \in C$ and $\mathcal{F}_{U_{p}}$ is the completion of $\mathcal{F}$ along $p$.

Proof. Recall that we have an affine covering $C=\operatorname{Spec} A \cup D_{+}(a)$ of the curve $C$, where $D_{+}(a)$ is defined in the proof of Theorem 3.3. On Spec $A$, we have a natural sheaf $W^{\sim}$, which is torsion free and of rank $r$. In order to construct a sheaf on $D_{+}(a)$, we need a torsion free module over $H^{0}\left(D_{+}(a), \mathcal{O}_{C}\right)$, where

$$
\begin{aligned}
H^{0}\left(D_{+}(a), \mathcal{O}_{C}\right) & =\left(\operatorname{gr} A\left[a^{-1}\right]\right)_{0} \\
& =\left\{a^{-\ell} v \mid \ell \geq 0, v \in A \text { and } \operatorname{ord}\left(a^{-\ell} v\right) \leq 0\right\} .
\end{aligned}
$$

So let us define

$$
W_{\infty}=\left\{a^{-\ell} w \mid \ell \geq 0, w \in W \text { and } \operatorname{ord}\left(a^{-\ell} w\right) \leq \nu\right\} \subset k((z)) .
$$

This determines a torsion free rank $r$ sheaf $W_{\infty}^{\sim}$ on $D_{+}(a)$. We want to define a sheaf $\mathcal{F}$ on $C$ by gluing $W^{\sim}$ on $\operatorname{Spec} A$ and $W_{\infty}^{\sim}$ on $D_{+}(a)$ together.

In order to do so, we have to show that there is a natural identity $W_{x}^{\sim}=W_{\infty, x}^{\sim}$ for every $x \in \operatorname{Spec} A \cap D_{+}(a)$. So let $x$ be a point of $\operatorname{Spec} A \cap D_{+}(a)$. Since $x \in \operatorname{Spec} A$, it corresponds to a prime ideal $I$ of $A$ such that $a \notin I$. As a point of $D_{+}(a), x$ corresponds also to a prime ideal

$$
I_{\infty}=\left\{a^{-\ell} f \mid \ell \geq 0, f \in I \text { and } \operatorname{ord}\left(a^{-\ell} f\right) \leq 0\right\}
$$

of $A_{\infty}=\left(\operatorname{gr} A\left[a^{-1}\right]\right)_{0}$. Now we have

$$
\begin{aligned}
W_{x}^{\sim} & =(A \backslash I)^{-1} W \\
& =\left\{v^{-1} w \mid v \in A \backslash I, w \in W\right\} \\
& \subset k((z)),
\end{aligned}
$$

and

$$
\begin{aligned}
W_{\infty, x}^{\sim} & =\left(A_{\infty} \backslash I_{\infty}\right)^{-1} W_{\infty} \\
& =\left\{\left(a^{-\ell} v\right)^{-1} a^{-m} w \mid \ell, m \geq 0, v \in A \backslash I, w \in W, \operatorname{ord}\left(a^{-\ell} v\right) \leq 0\right. \\
& \left.\quad \text { and } \operatorname{ord}\left(a^{-m} w\right) \leq \nu\right\} \\
& \subset k((z)) .
\end{aligned}
$$

Let $v^{-1} w \in W_{x}^{\sim}$. Since ord $a>0$, by taking $\ell>0$ large, we can make $\operatorname{ord}\left(a^{-\ell} v\right) \leq 0$ and $\operatorname{ord}\left(a^{-\ell} w\right) \leq \nu$. Thus $v^{-1} w=\left(a^{-\ell} v\right)^{-1} a^{-\ell} w \in W_{\infty, x}^{\sim}$, i.e. $W_{x}^{\sim} \subset W_{\infty, x}^{\sim}$. Conversely, let $\left(a^{-\ell} v\right)^{-1} a^{-m} w \in W_{\infty, x}^{\sim}$. If $\ell \geq m$, then $\left(a^{-\ell} v\right)^{-1} a^{-m} w=v^{-1}\left(a^{\ell-m} w\right) \in$ 
$W_{x}^{\sim}$, because $a^{\ell-m} w \in W$. On the other hand, if $\ell<m$, then $a^{m-\ell} v \in A \backslash I$ because $a \notin I$. Thus $\left(a^{-\ell} v\right)^{-1} a^{-m} w=\left(a^{m-\ell} v\right)^{-1} w \in W_{x}^{\sim}$, hence $W_{\infty, x}^{\sim} \subset W_{x}^{\sim}$. Therefore, we can define a sheaf $\mathcal{F}$ on $C$ by gluing $W^{\sim}$ and $W_{\infty}^{\sim}$ together on $\operatorname{Spec} A \cap D_{+}(a)$.

Since Coker $\gamma(\nu)_{W} \simeq V /\left(W+V^{(\nu)}\right)$ has finite dimension, there is an integer $N$ such that $W$ has an element of order $m$ for every $m \geq N$. Let $n \leq \nu$. Then there exists an integer $\ell$ such that

$$
\ell \cdot \operatorname{ord} a+n \geq N .
$$

Thus there is an element $w \in W$ of order $\ell \cdot \operatorname{ord} a+n$, and hence $\operatorname{ord}\left(a^{-\ell} w\right)=n \leq \nu$. This means that $W_{\infty}=H^{0}\left(D_{+}(a), \mathcal{F}\right)$ has an element of order $n$ for every $n \leq \nu$. Let $U_{p}$ be the formal completion of $C$ along $p$ as before and $\mathcal{F}_{U_{p}}$ be the formal completion of the sheaf $\mathcal{F}$ along $p$. Then we have

$$
\begin{aligned}
H^{0}\left(U_{p}, \mathcal{F}_{U_{p}}\right) & =H^{0}\left(D_{+}(a), \mathcal{F}\right) \underset{H^{0}\left(D_{+}(a), \mathcal{O}_{C}\right)}{\widehat{\otimes}} H^{0}\left(U_{p}, \mathcal{O}_{U_{p}}\right) \\
& =V^{(\nu)} \\
& \simeq H^{0}\left(U_{0}, \mathcal{O}_{U_{0}}(\nu)\right) \\
& \simeq H^{0}\left(U_{p}, \pi_{*} \mathcal{O}_{U_{0}}(\nu)\right) .
\end{aligned}
$$

This isomorphism determines an $\mathcal{O}_{U_{p}}$-module isomorphism

$$
\phi: \mathcal{F}_{U_{p}} \stackrel{\sim}{\longrightarrow} \pi_{*} \mathcal{O}_{U_{0}}(\nu) .
$$

Finally, since we have $W=H^{0}(C \backslash\{p\}, \mathcal{F})$, the same argument of Theorem 2.7 gives (3.9). This completes the proof.

Let us review what we have done so far. We started with a Schur pair $(A, W) \in$ $\mathcal{S}_{r}(\mu, \nu)$. Then we have constructed a curve $C$ (Theorem 3.3), a point $p(3.7)$, a torsion free sheaf $\mathcal{F}$ (Theorem 3.4), a local covering $\pi$ (3.8) and a local trivialization $\phi$ (Theorem 3.4). Thus we have obtained a quintet $(C, p, \mathcal{F}, \pi, \phi) \in \mathcal{Q}_{r}(\mu, \nu)$ out of the Schur pair $(A, W)$. Note that the above construction depends on the choice of the elements $a$ and $b$ used in the proof of Proposition 3.1. If we choose different elements $a^{\prime}$ and $b^{\prime}$, then we end up with another local parameter $y^{\prime}$, another local covering $\pi^{\prime}$ and another local trivialization $\phi^{\prime}$ of $\mathcal{F}$. But since we defined $\mathcal{F}$ so that we have $H^{0}\left(U_{p}, \mathcal{F}_{U_{p}}\right)=V^{(\nu)}$ canonically as above, the quintet $\left(C, p, \mathcal{F}, \pi^{\prime}, \phi^{\prime}\right)$ is identified with $(C, p, \mathcal{F}, \pi, \phi)$ by Definition 2.1. In other words, $(A, W) \in \mathcal{S}_{r}(\mu, \nu)$ defines a unique quintet $(C, p, \mathcal{F}, \pi, \phi)$ of $\mathcal{Q}_{r}(\mu, \nu)$. Thus we have obtained the inverse map of $\chi_{r, \mu, \nu}$, because it is obvious from the construction that

$$
\begin{aligned}
H^{0}\left(C \backslash\{p\}, \mathcal{O}_{C}\right) & =H^{0}\left(\operatorname{Spec} A, \mathcal{O}_{C}\right) \\
& =A
\end{aligned}
$$

and

$$
\begin{aligned}
H^{0}(C \backslash\{p\}, \mathcal{F}) & =H^{0}(\operatorname{Spec} A, \mathcal{F}) \\
& =W .
\end{aligned}
$$

Thus we obtain the following 
Theorem 3.5. Let $\mathcal{S}_{r}(\mu, \nu)$ be the set of all Schur pairs of rank $r$, index $\mu$ and level $\nu$, and let $\mathcal{Q}_{r}(\mu, \nu)$ denote the set of quintets of rank $r$, index $\mu$ and level $\nu$. Then there is a natural bijection

$$
\chi_{r, \mu, \nu}: \mathcal{Q}_{r}(\mu, \nu) \stackrel{\sim}{\longrightarrow} \mathcal{S}_{r}(\mu, \nu) .
$$

For a quintet $q=(C, p, \mathcal{F}, \pi, \phi)$, the corresponding Schur pair is given by

$$
\chi_{r, \mu, \nu}(q)=(A, W)=\left(\pi^{*}\left(H^{0}\left(C \backslash\{p\}, \mathcal{O}_{C}\right)\right), \phi\left(H^{0}(C \backslash\{p\}, \mathcal{F})\right)\right) .
$$

Definition 3.6. A contravariant functor $\chi_{(\nu)}: \mathcal{Q}(\nu) \rightarrow \mathcal{S}(\nu)$ is defined as follows:

(1) If $q=(C, p, \mathcal{F}, \pi, \phi) \in O b(\mathcal{Q}(\nu))$ is an element of $\mathcal{Q}_{r}(\mu, \nu)$, then we define

$$
\chi_{(\nu)}(q)=\chi_{r, \mu, \nu}(q) \in \mathcal{S}_{r}(\mu, \nu) .
$$

(2) For a morphism $(\beta, \psi):\left(C_{1}, p_{1}, \mathcal{F}_{1}, \pi_{1}, \phi_{1}\right) \rightarrow\left(C_{2}, p_{2}, \mathcal{F}_{2}, \pi_{2}, \phi_{2}\right)$, we define

$$
\chi_{(\nu)}((\beta, \psi))=(\alpha, \iota),
$$

where

$$
\alpha: \pi_{2}^{*}\left(H^{0}\left(C_{2} \backslash\left\{p_{2}\right\}, \mathcal{O}_{C_{2}}\right)\right) \longrightarrow \pi_{1}^{*}\left(H^{0}\left(C_{1} \backslash\left\{p_{1}\right\}, \mathcal{O}_{C_{1}}\right)\right)
$$

is given by the homomorphism

$$
H^{0}\left(C_{2} \backslash\left\{p_{2}\right\}, \mathcal{O}_{C_{2}}\right) \stackrel{\beta^{*}}{\longrightarrow} H^{0}\left(C_{1} \backslash\left\{p_{1}\right\}, \mathcal{O}_{C_{1}}\right)
$$

of cohomology groups associated with $\beta: C_{1} \rightarrow C_{2}$, and

$$
\iota: \phi_{2}\left(H^{0}\left(C_{2} \backslash\left\{p_{2}\right\}, \mathcal{F}_{2}\right)\right) \longrightarrow \phi_{1}\left(H^{0}\left(C_{1} \backslash\left\{p_{1}\right\}, \mathcal{F}_{1}\right)\right)
$$

is defined by the natural cohomology homomorphisms

$$
H^{0}\left(C_{2} \backslash\left\{p_{2}\right\}, \mathcal{F}_{2}\right) \stackrel{\psi}{\longrightarrow} H^{0}\left(C_{2} \backslash\left\{p_{2}\right\}, \beta_{*} \mathcal{F}_{1}\right) \stackrel{\beta^{*}}{\longrightarrow} H^{0}\left(C_{1} \backslash\left\{p_{1}\right\}, \mathcal{F}_{1}\right)
$$

Theorem 3.7. The functor $\chi_{(\nu)}: \mathcal{Q}(\nu) \rightarrow \mathcal{S}(\nu)$ makes these categories antiequivalent.

Proof. Only thing we have to show is that $\chi_{(\nu)}$ determines a well-defined bijective map

$$
\chi_{(\nu)}: \operatorname{Mor}_{\mathcal{Q}(\nu)}\left(q_{1}, q_{2}\right) \longrightarrow \operatorname{Mor}_{\mathcal{S}(\nu)}\left(\chi_{(\nu)}\left(q_{2}\right), \chi_{(\nu)}\left(q_{1}\right)\right)
$$


for every $q_{1}$ and $q_{2}$ in $\operatorname{Ob}(\mathcal{Q}(\nu))$. Let $A_{i}=\pi_{i}^{*}\left(H^{0}\left(C_{i} \backslash\left\{p_{i}\right\}, \mathcal{O}_{C_{i}}\right)\right)$ and $W_{i}=$ $\phi_{i}\left(H^{0}\left(C_{i} \backslash\left\{p_{i}\right\}, \mathcal{F}_{i}\right)\right), i=1,2$. We need to show that $\alpha: A_{2} \rightarrow A_{1}$ and $\iota: W_{2} \rightarrow W_{1}$ are inclusion maps. The commutative diagram

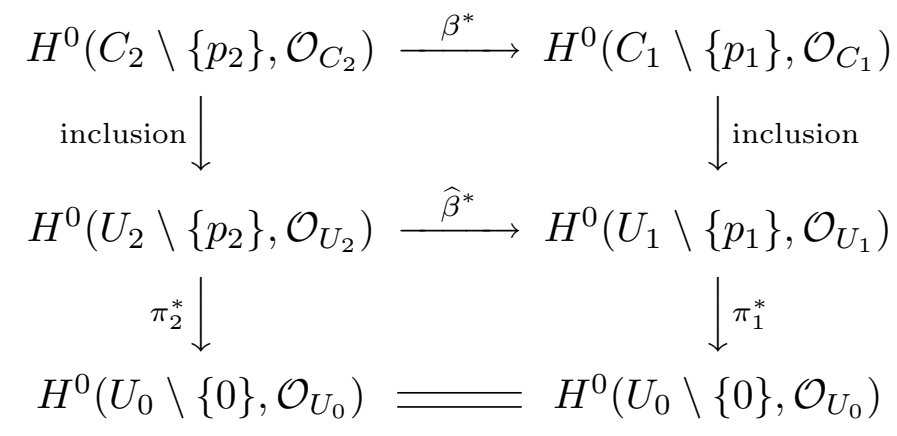

shows that $\alpha$ is an inclusion map, and the following diagram

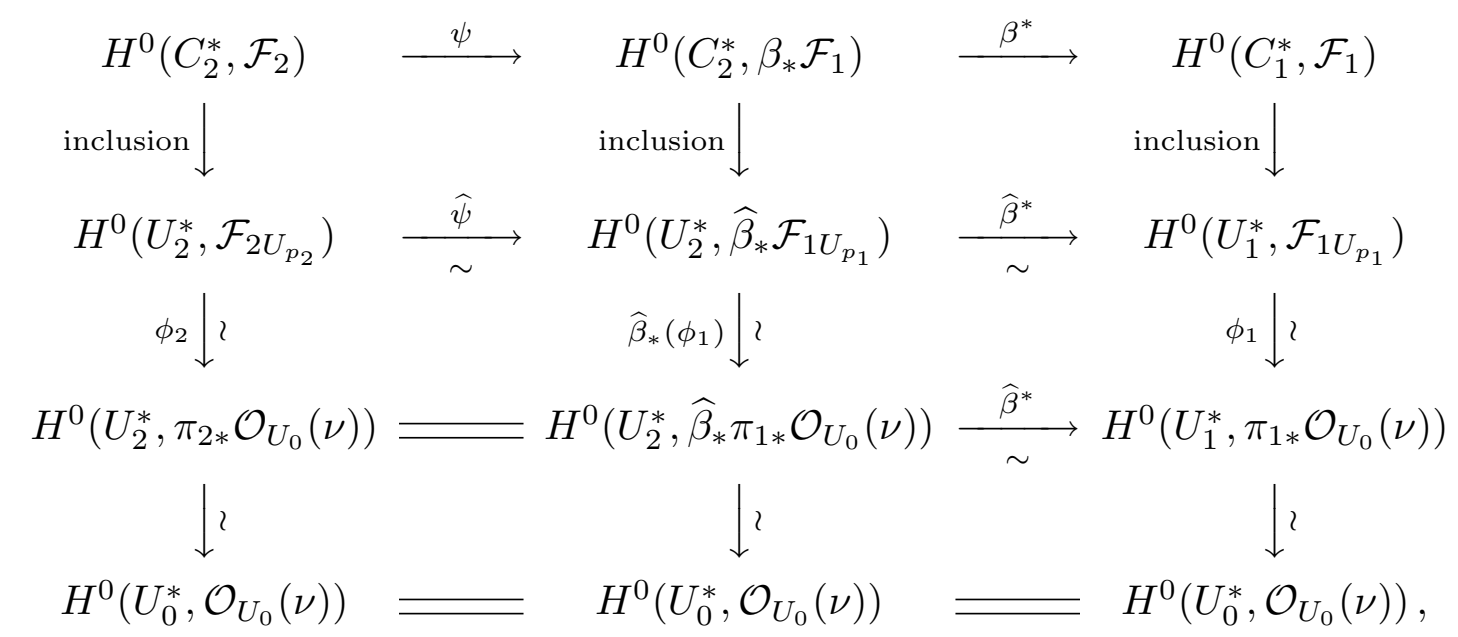

where $C_{i}^{*}=C_{i} \backslash\left\{p_{i}\right\}, U_{i}^{*}=U_{p_{i}} \backslash\left\{p_{i}\right\}$ and $U_{0}^{*}=U_{0} \backslash\{0\}$, shows that $\iota: W_{2} \rightarrow W_{1}$ is indeed an inclusion map.

In order to establish the bijectivity of $(3.10)$, let $(\alpha, \iota):\left(A_{2}, W_{2}\right) \rightarrow\left(A_{1}, W_{1}\right)$ be a morphism of the category $\mathcal{S}(\nu)$. Then the inclusion $\alpha: A_{2} \hookrightarrow A_{1}$ defines a covering map $\beta: C_{1} \rightarrow C_{2}$. It also makes $W_{1}$ an $A_{2}$-module and hence defines a sheaf $\beta_{*} \mathcal{F}_{1}$ on $C_{2}$. The inclusion map $\iota: W_{2} \hookrightarrow W_{1}$ then defines an injective homomorphism $\psi: \mathcal{F}_{2} \rightarrow \beta_{*} \mathcal{F}_{1}$.

Since $\beta$ maps the affine subscheme Spec $A_{1}$ of $C_{1}$ to Spec $A_{2}$ surjectively, we have $\beta\left(p_{1}\right)=p_{2}$, which verifies (2.1). If we choose the key element $a$ of (3.3) from $A_{2}$, then $\alpha$ gives a natural inclusion map

$$
\begin{aligned}
\left(\operatorname{gr} A_{2}\left[a^{-1}\right]\right)_{0} & =\left\{a^{-\ell} v \mid \ell \geq 0, v \in A_{2} \text { and } \operatorname{ord}\left(a^{-\ell} v\right) \leq 0\right\} \\
& \hookrightarrow\left\{a^{-\ell} v \mid \ell \geq 0, v \in A_{1} \text { and } \operatorname{ord}\left(a^{-\ell} v\right) \leq 0\right\} \\
& =\left(\operatorname{gr} A_{1}\left[a^{-1}\right]\right)_{0} \\
& \subset k[[z]]
\end{aligned}
$$


which defines a surjective morphism $\widehat{\beta}: U_{p_{1}} \longrightarrow U_{p_{2}}$. It satisfies $(2.2)$ because of this construction.

Similarly, we have

$$
\begin{aligned}
W_{2, \infty} & =\left\{a^{-\ell} w \mid \ell \geq 0, w \in W_{2} \text { and } \operatorname{ord}\left(a^{-\ell} w\right) \leq \nu\right\} \\
& \hookrightarrow\left\{a^{-\ell} w \mid \ell \geq 0, w \in W_{1} \text { and } \operatorname{ord}\left(a^{-\ell} w\right) \leq \nu\right\} \\
& =W_{1, \infty} \\
& \subset k((z)) .
\end{aligned}
$$

The above map determines a homomorphism $\widehat{\psi}: \mathcal{F}_{2 U_{p_{2}}} \longrightarrow \widehat{\beta}_{*} \mathcal{F}_{1 U_{p_{1}}}$ which satisfies (2.3). Thus we have constructed a morphisn $(\beta, \psi)$ between the corresponding objects in $\mathcal{Q}(\nu)$. This completes the proof.

Proposition 3.8. Let $(A, W)$ be a Schur pair of index zero. Then the sheaf $\mathcal{F}$ of the corresponding quintet is semistable if $W$ is a point of the big cell of the Grassmannian.

Proof. If $W \in G^{+}(0, \nu)$, then the sheaf $\mathcal{F}$ satisfies

$$
H^{0}(C, \mathcal{F})=H^{1}(C, \mathcal{F})=0
$$

because of the isomorphism (3.9) of Theorem 3.4. Let $0 \rightarrow \mathcal{L} \rightarrow \mathcal{F}$ be a torsion free subsheaf of $\mathcal{F}$. Then by the Riemann-Roch Theorem, we have

$$
\begin{aligned}
\frac{\operatorname{deg} \mathcal{L}}{\operatorname{rank} \mathcal{L}} & =\frac{\operatorname{dim}_{k} H^{0}(C, \mathcal{L})-\operatorname{dim}_{k} H^{1}(C, \mathcal{L})+(g-1) \cdot \operatorname{rank} \mathcal{L}}{\operatorname{rank} \mathcal{L}} \\
& =g-1-\frac{\operatorname{dim}_{k} H^{1}(C, \mathcal{L})}{\operatorname{rank} \mathcal{L}} \\
& \leq g-1 \\
& =\frac{\operatorname{dim}_{k} H^{0}(C, \mathcal{F})-\operatorname{dim}_{k} H^{1}(C, \mathcal{F})+(g-1) \cdot \operatorname{rank} \mathcal{F}}{\operatorname{rank} \mathcal{F}} \\
& =\frac{\operatorname{deg} \mathcal{F}}{\operatorname{rank} \mathcal{F}},
\end{aligned}
$$

where $g$ is the genus of the curve $C$. Therefore, $\mathcal{F}$ is semistable.

\section{Pseudo-differential operators acting on the Grassmannians.}

We have established equivalence of the categories $\mathcal{Q}(\nu)$ and $\mathcal{S}(\nu)$ in the last section. When we defined $\mathcal{Q}(\nu)$, we noted that the thickened category $\overline{\mathcal{Q}}(\nu)$ of Definition 2.3 is more natural from the geometric point of view because of its coordinate-free nature. Now we can ask the following: what would be the corresponding thickened category $\overline{\mathcal{S}}(\nu)$ so that our functor $\chi_{(\nu)}$ extends to an equivalence 
of thickened categories? Certainly, $\overline{\mathcal{S}}(\nu)$ should have exactly the same objects as $\mathcal{S}(\nu)$ does. Then what would be a natural morphism among Schur pairs other than inclusions?

The answer comes from an unexpected direction-from algebraic theory of pseudodifferential operators. In this section, we investigate necessary accounts on pseudodifferential operators and define the thickened category of Schur pairs. Then we prove the equivalence of $\overline{\mathcal{Q}}(\nu)$ and $\overline{\mathcal{S}}(\nu)$.

Let us start with defining the function space we are going to use. So let

$$
R=k[[x]]
$$

be the ring of formal power series in one variable $x$ with coefficients in $k$ and let $\partial=\frac{d}{d x}$ be the differentiation with respect to $x$. The set of all formal ordinary pseudo-differential operators with coefficients in $R$ is defined by

$$
E=\left\{\sum_{n \in \mathbb{Z}} f_{n} \partial^{n} \mid f_{n} \in R \text { and } f_{n}=0 \text { for all } n \gg 0\right\}
$$

Every element of $E$ has a form

$$
P=a_{0} \partial^{N}+a_{1} \partial^{N-1}+a_{2} \partial^{N-2}+\cdots+a_{N}+a_{N+1} \partial^{-1}+\cdots
$$

for some finite integer $N$ depending on $P$. We say that $P$ is of order $N$ if $a_{0} \neq 0$ and monic if $a_{0}=1$.

From the definition of (4.2), $E$ has a natural structure of left $R$-module. It also has a filtration defined by the order of operators

$$
\cdots \supset E^{(n+1)} \supset E^{(n)} \supset E^{(n-1)} \supset \cdots,
$$

where

$$
E^{(n)}=\left\{\sum_{m \in \mathbb{Z}} f_{m} \partial^{m} \mid f_{m} \in R \text { and } f_{m}=0 \text { for all } m>n\right\} .
$$

By defining $\left\{E^{(n)}\right\}_{n \in \mathbb{Z}}$ as a basis for open sets, $E$ becomes a complete topological space because of

$$
\bigcup_{n \in \mathbb{Z}} E^{(n)}=E \quad \text { and } \bigcap_{n \in \mathbb{Z}} E^{(n)}=\{0\}
$$

For a function $f \in R$, we denote by $f^{(i)}$ the $i$-th derivative of $f$ with respect to $x$. The Leibniz rule

$$
\partial^{n} \cdot f=\sum_{i=0}^{\infty}\left(\begin{array}{l}
n \\
i
\end{array}\right) f^{(i)} \partial^{n-i}
$$

holds for an arbitrary integer $n \in \mathbb{Z}$ if we define

$$
\left(\begin{array}{c}
n \\
i
\end{array}\right)=\frac{\Gamma(n+1)}{\Gamma(i+1) \Gamma(n-i+1)}=\frac{n \cdot(n-1) \cdot \cdots \cdot(n-i+1)}{i \cdot(i-1) \cdot \cdots \cdot 1} .
$$


Therefore, we can introduce an associative algebra structure in $E$ as follows: Let $P=\sum_{m=0}^{\infty} a_{m} \partial^{M-m}$ and $Q=\sum_{n=0}^{\infty} b_{n} \partial^{N-n}$ be operators in $E$. Then we define

$$
\begin{aligned}
& P \cdot Q=\sum_{m=0}^{\infty} a_{m} \partial^{M-m} \cdot \sum_{n=0}^{\infty} b_{n} \partial^{N-n} \\
& =\sum_{m=0}^{\infty} \sum_{n=0}^{\infty} \sum_{i=0}^{\infty}\left(\begin{array}{c}
M-m \\
i
\end{array}\right) a_{m} b_{n}^{(i)} \partial^{M+N-m-n-i} \\
& =\sum_{\ell=0}^{\infty}\left(\sum_{m=0}^{\ell} \sum_{i=0}^{\ell-m}\left(\begin{array}{c}
M-m \\
i
\end{array}\right) a_{m} b_{\ell-m-i}^{(i)}\right) \partial^{M+N-\ell} .
\end{aligned}
$$

Since the coefficient of $\partial^{M+N-\ell}$ is a finite sum for every $\ell \geq 0, P \cdot Q$ is a well-defined element in $E$.

An operator $P \in E$ is said to be in the right normal form if $P=\sum_{m=0}^{\infty} a_{m} \partial^{M-m}$, and in the left normal form if $P=\sum_{n=0}^{\infty} \partial^{M-n} \cdot b_{n}$. The adjoint Leibniz rule

$$
f \partial^{n}=\sum_{i=0}^{\infty}(-1)^{i}\left(\begin{array}{c}
n \\
i
\end{array}\right) \partial^{n-i} \cdot f^{(i)}
$$

which can be easily checked, gives a way of translating the right normal form to the left normal form and vice versa. For example, since

$$
\begin{aligned}
\sum_{m=0}^{\infty} a_{m} \partial^{M-m} & =\sum_{m=0}^{\infty} \sum_{i=0}^{\infty}(-1)^{i}\left(\begin{array}{c}
M-m \\
i
\end{array}\right) \partial^{M-m-i} \cdot a_{m}^{(i)} \\
& =\sum_{n=0}^{\infty} \partial^{M-n} \cdot\left\{\sum_{i=0}^{n}(-1)^{i}\left(\begin{array}{c}
M-n+i \\
i
\end{array}\right) a_{n-i}^{(i)}\right\},
\end{aligned}
$$

we have

$$
b_{n}=\sum_{i=0}^{n}(-1)^{i}\left(\begin{array}{c}
M-n+i \\
i
\end{array}\right) a_{n-i}^{(i)} .
$$

In particular, we have $b_{0}=a_{0}$ and $b_{M+1}=a_{M+1}$. This means that the order, monicness and the residue (the coefficient of $\partial^{-1}$ ) of an operator do not depend on the expression (right or left normal form) of the operator. Thus the filtraton (4.4) does not depend on the expression either.

The ring $D$ of ordinary differential operators is defined by

$$
D=\left\{\sum_{n=0}^{\infty} f_{n} \partial^{n} \mid f_{n} \in R \text { and } f_{n}=0 \text { for all } n \gg 0\right\},
$$


which is a subring of $E$. Note that if $P=\sum_{n \in \mathbb{Z}} f_{n} \partial^{n}=\sum_{m \in \mathbb{Z}} \partial^{m} \cdot g_{m}$, then $f_{n}=0$ for all $n<0$ if and only if $g_{m}=0$ for all $m<0$. Thus the definition of $D$ does not depend on the choice of the expression of operators. The filtration (4.3) of $E$ induces a filtration

$$
\cdots \supset D^{(n+1)} \supset D^{(n)} \supset D^{(n-1)} \supset \cdots \supset D^{(0)}=R
$$

of $D$, where we define $D^{(n)}=D \cap E^{(n)}$.

Now let us consider the left maximal ideal $E x$ of $E$ generated by $x \in E$ and let $\rho: E \longrightarrow E / E x$ denote the natural projection. In the left normal form, we have

$$
\rho\left(\sum_{n \in \mathbb{Z}} \partial^{n} \cdot f_{n}(x)\right)=\sum_{n \in \mathbb{Z}} \partial^{n} \cdot f_{n}(0)=\sum_{n \in \mathbb{Z}} f_{n}(0) \partial^{n} .
$$

Therefore, we have an equality $E / E x=k\left(\left(\partial^{-1}\right)\right)$. At this stage, we introduce an identification

$$
\partial^{-1}=z
$$

Then $k\left(\left(\partial^{-1}\right)\right)=k((z))=V$, hence we obtain a $k$-linear map

$$
\rho: E \longrightarrow V=E / E x
$$

Since $\rho\left(\sum_{n=0}^{\infty} \partial^{N-n} \cdot f_{n}(x)\right)=\sum_{n=0}^{\infty} f_{n}(0) z^{-N+n} \in V$, we have $\rho\left(E^{(n)}\right)=V^{(n)}$. Using the above projection map, we can define the $E$-action on the vector space $V=$ $E / E x$.

Definition 4.1. Let $P \in E$ be an arbitrary pseudo-differential operator and let $v \in V$ be an arbitrary vector. Since $V=E / E x$, there is an operator $Q \in E$ such that $\rho(Q)=v$. We define a k-linear map $P: V \longrightarrow V$ by

$$
P v=\rho(P Q) \in V \text {. }
$$

We have to check the well-definedness of this map. So let $Q^{\prime} \in E$ be another operator such that $\rho\left(Q^{\prime}\right)=\rho(Q)=v$. Then

$$
\begin{aligned}
\rho\left(P Q^{\prime}\right) & =\rho\left(P Q^{\prime}+P\left(Q-Q^{\prime}\right)\right) \\
& =\rho(P Q)
\end{aligned}
$$

because $P\left(Q-Q^{\prime}\right) \in E x$. Thus $P: V \rightarrow V$ is well-defined. If $P \in E$ is invertible, then $P^{-1}: V \longrightarrow V$ gives the inverse map of $P: V \rightarrow V$, because

$$
P^{-1} \rho(P Q)=\rho\left(P^{-1} P Q\right)=\rho(Q) .
$$

Therefore, an invertible element $P$ gives an automorphism of $V$. Moreover, if $P$ is of order zero, then it induces an automorphism $P: V^{(n)} \stackrel{\sim}{\longrightarrow} V^{(n)}$ for every $n \in \mathbb{Z}$, and hence a homeomorphism of $V$ with respect to its topology. 
Proposition 4.2. Let $P \in E$ be an invertible operator of order zero and let $P$ : $V \stackrel{\sim}{\rightarrow} V$ be the homeomorphism defined above. Then $P$ induces an automorphism

$$
P: G(\mu, \nu) \stackrel{\sim}{\longrightarrow} G(\mu, \nu)
$$

of the Grassmannian of index $\mu$ and level $\nu$ for every $\mu, \nu \in \mathbb{Z}$.

Proof. Let $W \in G(\mu, \nu)$. Since $P: V \stackrel{\sim}{\rightarrow} V$ is a homeomorphism, $P W \subset V$ is a closed subspace. Because $P V=V$ and $P V^{(\nu)}=V^{(\nu)}$, we have

$$
\begin{aligned}
\operatorname{Ker} \gamma(\nu)_{W} & =W \cap V^{(\nu)} \\
& \simeq P W \cap P V^{(\nu)} \\
& =P W \cap V^{(\nu)} \\
& =\operatorname{Ker} \gamma(\nu)_{P W}
\end{aligned}
$$

Similarly,

$$
\begin{aligned}
\operatorname{Coker} \gamma(\nu)_{W} & =V /\left(W+V^{(\nu)}\right) \\
& \simeq P V /\left(P W+P V^{(\nu)}\right) \\
& =V /\left(P W+V^{(\nu)}\right) \\
& =\operatorname{Coker} \gamma(\nu)_{P W} .
\end{aligned}
$$

Therefore, Index $\gamma(\nu)_{W}=\operatorname{Index} \gamma(\nu)_{P W}$. Thus $P$ maps $G(\mu, \nu)$ into itself, and its inverse is given by $P^{-1}$. This completes the proof.

More general element of $E$ does not give an automorphism of the Grassmannians, but it produces a vector field on them. In order to see this, let us recall that the group $\operatorname{Hom}(W, V / W)$ of continuous homomorphisms represents the tangent space of the Grassmannian at the given point $W$. Now, for every pseudo-differential operator $P \in E$, we define

$$
\Phi_{W}(P): W \hookrightarrow V \stackrel{P}{\longrightarrow} V \rightarrow V / W
$$

which is obviously a continuous homomorphism of $W$ into $V / W$. Thus $\Phi_{W}(P) \in$ $T_{W} G(\mu, \nu)$ and we can identify $\Phi(P)$ a vector field on the Grassmannian. We say that the algebra $E$ acts on the Grassmannians infinitesimally, which means that every element of $E$ determines a vector field on the Grassmannians.

In Section 6, we will see that the action of the pseudo-differential operators of constant coefficients recovers the entire KP system. From this Grassmannian point of view, it turns out to be very easy to see why every integral manifold of the action of constant coefficient operators has a natural structure of the Jacobian variety of an algebraic curve. 
The other interesting class of action is the Virasoro action on the Grassmannian. Let $\mathcal{H}=k\left(\left(\partial^{-1}\right)\right) \cdot x$. It has a natural structure of Lie algebra by the operator bracket. Under the Fourier transformation, $\mathcal{H}$ becomes $k((z)) \frac{d}{d z}$, which is the Lie algebra of formal vector fields on $U_{0}$ with finite order poles at the origin. The Virasoro action on the Grassmannians was studied by many authors for the case of $k=\mathbb{C}$. See for example, [ADKP], [BS], [KNTY], and [Wit].

Here, let us make a remark on the $V$-action on $V$ itself. Since $V=k((z)), V$ acts on $V$ by multiplication. On the other hand, the identification $z=\partial^{-1}$ makes $V$ the set of pseudo-differential operators with constant coefficients. Thus $V=$ $k\left(\left(\partial^{-1}\right)\right) \subset E$ acts on $V=E / E x$ by the left multiplication of pseudo-differential operators given in Definition 4.1. The important point we have here is that both actions of $V$ on $V$ described above have exactly the same results. When we defined the Schur pair $(A, W)$ in Definition 1.2, we considered $A$ and $W$ subspaces of $V=$ $k((z))$. Certainly, $W$ is a point of the Grassmannian, and hence $W$ is a subspace of $V=E / E x$. However, since $A$ acts on $W$, from functorial point of view, it is more natural to regard

$$
A \subset k\left(\left(\partial^{-1}\right)\right) \subset E .
$$

Therefore, whenever we use a $\operatorname{Schur}$ pair $(A, W)$ from now on, we understand that $A \subset k\left(\left(\partial^{-1}\right)\right) \subset E$ and $W \subset V=E / E x$.

Definition 4.3. A pseudo-differential operator $T \in E$ is said to be admissible if it is an invertible operator of order zero such that $T \cdot \partial \cdot T^{-1} \in k\left(\left(\partial^{-1}\right)\right)$. The set of all admissible operators is denoted by $\Gamma_{a}$.

Every admissible operator $T$ has the following form:

$$
\begin{aligned}
T & =c_{0} T_{0} \cdot e^{c_{1} x} \\
T_{0} & =1+a_{1}(x) \partial^{-1}+a_{2}(x) \partial^{-2}+\cdots
\end{aligned}
$$

where $0 \neq c_{0} \in k, c_{1} \in k$ and $a_{n}(x)$ is a polynomial in $x$ of degree less than or equal to $n$.

Definition 4.4. We define the thickened category $\overline{\mathcal{S}}(\nu)$ of Schur pairs as follows:

(1) $O b(\overline{\mathcal{S}}(\nu))=O b(\mathcal{S}(\nu))$.

(2) A morphism $T:\left(A_{2}, W_{2}\right) \longrightarrow\left(A_{1}, W_{1}\right)$ consists of twisted inclusions

$$
T A_{2} T^{-1} \hookrightarrow A_{1} \quad \text { and } \quad T W_{2} \hookrightarrow W_{1},
$$

where $T$ is an arbitrary admissible operator.

The following lemma is a key lemma to prove equivalence of thickened categories. From now on, we have to assume that our field $k$ has characteristic zero. 
Lemma 4.5. Let $v \in k\left(\left(\partial^{-1}\right)\right)$ be an arbitrary monic element of order -1 , where $k$ is a field of characteristic zero. Then there exists an admissible operator $T \in \Gamma_{a}$ such that $T \partial^{-1} T^{-1}=v$.

This is an easy consequence of Lemma 7.5.

Theorem 4.6. Let $k$ be a field of characteristic zero. Then the functor $\chi_{(\nu)}$ of Definition 3.6 induces a new functor

$$
\bar{\chi}_{(\nu)}: \overline{\mathcal{Q}}(\nu) \longrightarrow \overline{\mathcal{S}}(\nu)
$$

which makes these categories anti-equivalent.

Proof. Let us start with a morphism $(\beta, \psi): q_{1} \rightarrow q_{2}$ between two quintets $q_{i}=$ $\left(C_{i}, p_{i}, \mathcal{F}_{i}, \pi_{i}, \phi_{i}\right) \in O b(\overline{\mathcal{Q}}(\nu))$. We have an automorphism $h: U_{0} \stackrel{\sim}{\rightarrow} U_{0}$ and a ring automorphism

$$
k[[z]] \ni f(z) \stackrel{\bar{h}}{\longmapsto} f(\bar{h}(z)) \in k[[z]]
$$

of (2.5) which is given by $\bar{h}(z)=z+a_{2} z^{2}+a_{3} z^{3}+\cdots$. By the identification $z=\partial^{-1}$, we have $\bar{h}\left(\partial^{-1}\right)=\partial^{-1}+a_{2} \partial^{-2}+a_{3} \partial^{-3}+\cdots \in k\left(\left(\partial^{-1}\right)\right)$. Because of Lemma 4.5, there is an admissible operator $T_{1} \in \Gamma_{a}$ such that

$$
T_{1} \partial^{-1} T_{1}^{-1}=\bar{h}\left(\partial^{-1}\right) .
$$

The ring automorphism $\bar{h}: k[[z]] \rightarrow k[[z]]$ extends to a field automorphism $\bar{h}:$ $k((z)) \rightarrow((z))$ in an obvious way. Thus

$$
\begin{aligned}
k\left(\left(\partial^{-1}\right)\right) \ni f\left(\partial^{-1}\right) \longmapsto f\left(\bar{h}\left(\partial^{-1}\right)\right) & =f\left(T_{1} \partial^{-1} T_{1}^{-1}\right) \\
& =T_{1} f\left(\partial^{-1}\right) T_{1}^{-1} \\
& \in k\left(\left(\partial^{-1}\right)\right) .
\end{aligned}
$$

The $\mathcal{O}_{U_{0}}$-module isomorphism $\xi: \mathcal{O}_{U_{0}}(\nu) \stackrel{\sim}{\rightarrow} h_{*} \mathcal{O}_{U_{0}}(\nu)$ of $(2.7)$ is given by the multiplication of a single invertible element $\xi \in \mathcal{O}_{U_{0}}^{\times}$. Using the coordinate $z$ on $U_{0}$, we can expand $\xi=\sum_{i=0}^{\infty} c_{i} z^{i}, c_{0} \neq 0$. It determines an admissible operator $T_{2}=$ $\sum_{i=0}^{\infty} c_{i} \partial^{-i}$. Since it is an operator having only constant coefficients, $T_{2} A T_{2}^{-1}=A$ for every subset $A$ of $k\left(\left(\partial^{-1}\right)\right)$.

Now, let $A_{i}=\pi_{i}^{*}\left(H^{0}\left(C_{i} \backslash\left\{p_{i}\right\}, \mathcal{O}_{C_{i}}\right)\right)$ and $W_{i}=\phi_{i}\left(H^{0}\left(C_{i} \backslash\left\{p_{i}\right\}, \mathcal{F}_{i}\right)\right), i=1,2$. Since we have 


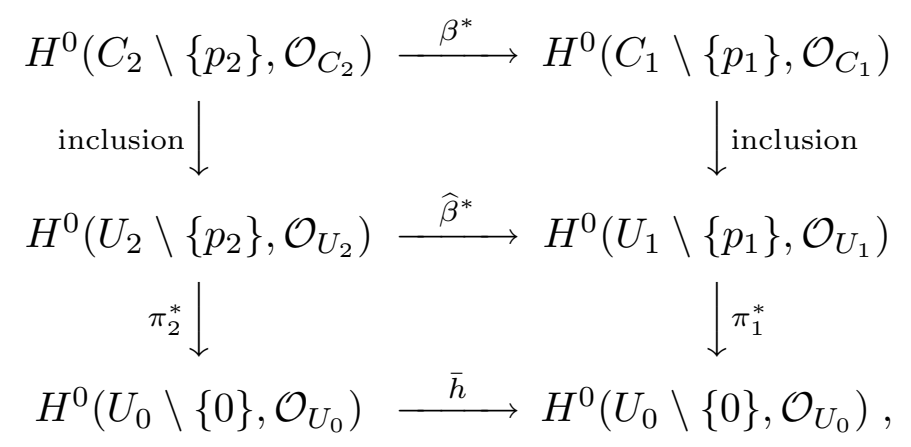

we obtain

$$
\begin{aligned}
T_{1} T_{2} A_{2} T_{2}^{-1} T_{1}^{-1} & =T_{1} A_{2} T_{1}^{-1} \\
& =\bar{h}\left(A_{2}\right) \\
& =\bar{h} \pi_{2}^{*}\left(H^{0}\left(C_{2} \backslash\left\{p_{2}\right\}, \mathcal{O}_{C_{2}}\right)\right) \\
& \subset \pi_{1}^{*}\left(H^{0}\left(C_{1} \backslash\left\{p_{1}\right\}, \mathcal{O}_{C_{1}}\right)\right) \\
& =A_{1}
\end{aligned}
$$

On the other hand, since $\pi_{2 *} h_{*} \mathcal{O}_{U_{0}}(\nu)=\widehat{\beta}_{*} \pi_{1 *} \mathcal{O}_{U_{0}}(\nu)$, we have

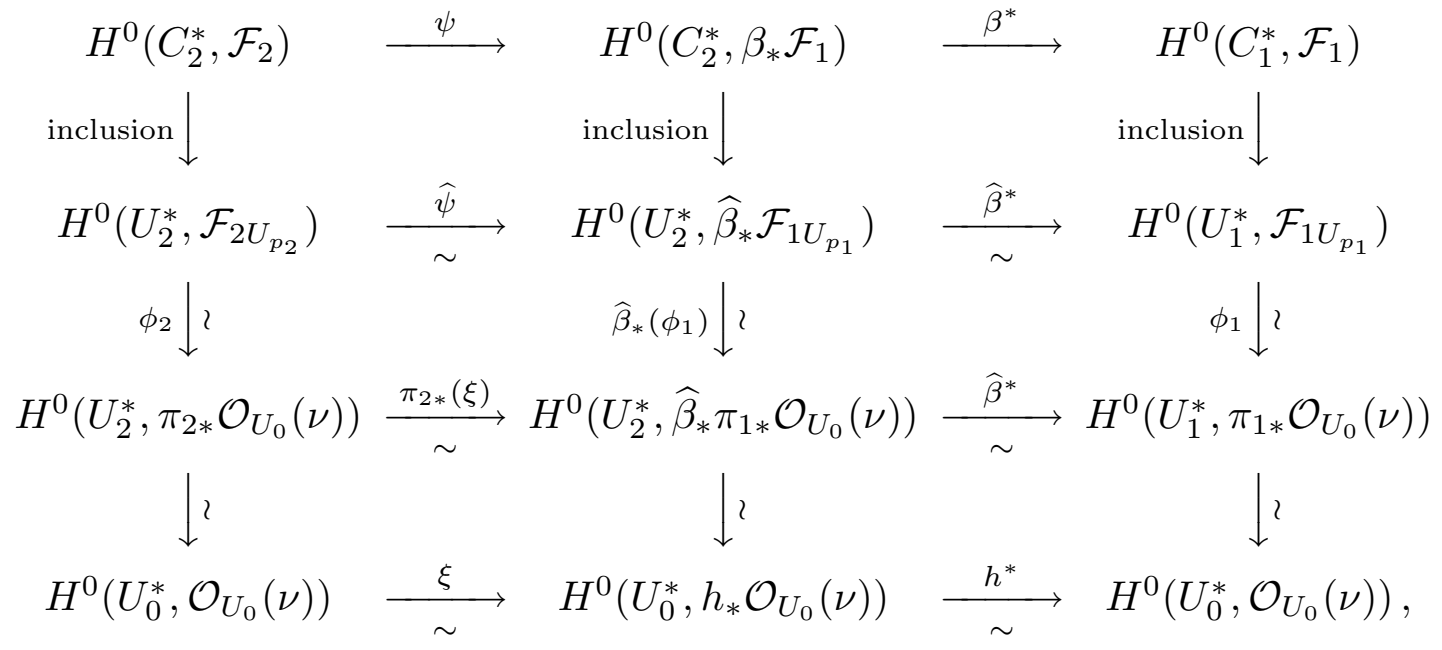

where $C_{i}^{*}=C_{i} \backslash\left\{p_{i}\right\}, U_{i}^{*}=U_{p_{i}} \backslash\left\{p_{i}\right\}$ and $U_{0}^{*}=U_{0} \backslash\{0\}$.

Since the isomorphism $h^{*}$ in the above diagram is a homomorphism from an

$$
H^{0}\left(U_{0}^{*}, \mathcal{O}_{U_{0}}\right) \text {-module } \quad H^{0}\left(U_{0}^{*}, h_{*} \mathcal{O}_{U_{0}}(\nu)\right)
$$

to an

$$
\bar{h}\left(H^{0}\left(U_{0}^{*}, \mathcal{O}_{U_{0}}\right)\right) \text {-module } \quad H^{0}\left(U_{0}^{*}, \mathcal{O}_{U_{0}}(\nu)\right),
$$


it is completely determined by its image $h^{*}(1)$ of the generator

$$
1 \in H^{0}\left(U_{0}^{*}, h_{*} \mathcal{O}_{U_{0}}(\nu)\right) .
$$

Every element of this cohomology is of the form $f(z) \cdot 1$, where $f(z) \in H^{0}\left(U_{0}^{*}, \mathcal{O}_{U_{0}}\right)$. Hence

$$
\begin{aligned}
h^{*}(f(z) \cdot 1) & =f(\bar{h}(z)) \cdot h^{*}(1) \\
& =T_{1} \cdot f\left(\partial^{-1}\right) \cdot T_{1}^{-1} \cdot h^{*}(1) .
\end{aligned}
$$

Therefore, we conclude that $h^{*}=T_{1}$, because of the following consistency:

$$
\begin{aligned}
h^{*}(f(z) \cdot 1) & =T_{1} \cdot f\left(\partial^{-1}\right) \cdot T_{1}^{-1} \cdot T_{1} \cdot 1 \\
& =T_{1} \cdot f\left(\partial^{-1}\right) \\
& =T_{1} \cdot f(z) .
\end{aligned}
$$

Thus we have

$$
T_{1} T_{2}\left(W_{2}\right)=h^{*} \xi \phi_{2}\left(H^{0}\left(C_{2}^{*}, \mathcal{F}_{2}\right)\right) \subset \phi_{1}\left(H^{0}\left(C_{1}^{*}, \mathcal{F}_{1}\right)\right)=W_{1}
$$

Let $T=T_{1} T_{2}$. Then it is an admissible operator and we have $T A_{2} T^{-1} \subset A_{1}$ and $T W_{2} \subset W_{1}$. Hence we have constructed a morphism

$$
T:\left(A_{2}, W_{2}\right) \longrightarrow\left(A_{1}, W_{1}\right) \text {. }
$$

Conversely, let $T:\left(A_{2}, W_{2}\right) \longrightarrow\left(A_{1}, W_{1}\right)$ be a morphism between Schur pairs defined by an admissible opertor $T \in \Gamma_{a}$. It means that we have

$$
T A_{2} T^{-1} \subset A_{1} \text { and } T W_{2} \subset W_{1} .
$$

Let $C_{i}$ be the complete algebraic curve defined by $A_{i}$ and $\mathcal{F}_{i}$ be the torsion free sheaf corresponding to $W_{i}, i=1,2$. Note that $W_{1}$ has a natural $T A_{2} T^{-1}$-module structure. Thus the inclusions of (4.8) define a morphism $\beta: C_{1} \longrightarrow C_{2}$ and a sheaf homomorphism $\psi: \mathcal{F}_{2} \longrightarrow \beta_{*} \mathcal{F}_{1}$.

Now, since $T$ is admissible, we have

$$
T k[[z]] T^{-1}=T k\left[\left[\partial^{-1}\right]\right] T^{-1} \stackrel{\sim}{\longrightarrow} k\left[\left[\partial^{-1}\right]\right]=k[[z]]
$$

and which gives a formal scheme automorphism

$$
h: U_{0} \stackrel{\sim}{\longrightarrow} U_{0} .
$$

Moreover, $T$ gives an isomorphism between $k\left(\left(\partial^{-1}\right)\right)$-module $V$ and $T k\left(\left(\partial^{-1}\right)\right) T^{-1}$ module $T V$. Since $V$ is generated by the identity element $1 \in V$ as a $k\left(\left(\partial^{-1}\right)\right)$ module, $T: V \rightarrow V$ is determined by its image $T \cdot 1$. Let

$$
\xi=T \cdot 1=\rho(T) \in V=k[[z]] .
$$


Then $\xi$ is an invertible element of order zero and hence $\xi \in \mathcal{O}_{U_{0}}^{\times}$. Every element of $V$ is uniquely expressed as $f\left(\partial^{-1}\right) \cdot 1$, where $f\left(\partial^{-1}\right) \in k\left(\left(\partial^{-1}\right)\right)$. We have

$$
\begin{aligned}
T \cdot\left(f\left(\partial^{-1}\right) \cdot 1\right) & =T f\left(\partial^{-1}\right) T^{-1} \cdot(T \cdot 1) \\
& =f\left(T \partial^{-1} T^{-1}\right) \cdot \xi \\
& =f(\bar{h}(z)) \cdot \xi .
\end{aligned}
$$

It is easy to check that $h$ satisfies (2.5) and (2.6), and $\xi$ defines an $\mathcal{O}_{U_{0}}$-module isomorphism

$$
\xi: \mathcal{O}_{U_{0}}(\nu) \stackrel{\sim}{\longrightarrow} h_{*} \mathcal{O}_{U_{0}}(\nu)
$$

which satisfies (2.7). This completes the proof.

We say that $\left(A_{1}, W_{1}\right)$ and $\left(A_{2}, W_{2}\right)$ are isomorphic if there is an admissible operator $T$ such that $A_{1}=T A_{2} T^{-1}$ and $W_{1}=T W_{2}$.

Lemma 4.7. Let $T \in \Gamma_{a}$ be an arbitrary admissible operator. Then $T$ induces a bijection

$$
T: \mathcal{S}_{r}(\mu, \nu) \stackrel{\sim}{\longrightarrow} \mathcal{S}_{r}(\mu, \nu)
$$

defined by $T \cdot(A, W)=\left(T A T^{-1}, T W\right)$.

Proof. Since $T$ is invertible and $\left(T^{-1} T A T^{-1} T, T^{-1} T W\right)=(A, W)$, only thing we have to check is that $T \cdot(A, W) \in \mathcal{S}_{r}(\mu, \nu)$ for every $(A, W) \in \mathcal{S}_{r}(\mu, \nu)$. Since $T \partial T^{-1} \in k\left(\left(\partial^{-1}\right)\right)$, we have

$$
T A T^{-1} \subset T k\left(\left(\partial^{-1}\right)\right) T^{-1} \subset k\left(\left(\partial^{-1}\right)\right)
$$

Certainly, $\left(T A T^{-1}\right) T W=T A W \subset T W$. Let us compute the rank of $T A T^{-1}$. Since ord $P=\operatorname{ord}\left(T P T^{-1}\right)$ for every $P \in E$, we have $\{\operatorname{ord} a \mid a \in A\}=\{\operatorname{ord} b \mid b \in$ $\left.T A T^{-1}\right\}$. Therefore, $\operatorname{rank} A=r=\operatorname{rank} T A T^{-1}$. Finally, since ord $T=0, T W$ is an element of the Grassmannian $G(\mu, \nu)$ because of Proposition 4.2. Thus we established that $T \cdot(A, W) \in \mathcal{S}_{r}(\mu, \nu)$. This completes the proof.

The set of isomorphism classes of Schur pairs of level $\mu$ and level $\nu$ is denoted by $\mathcal{S}(\mu, \nu) / \Gamma_{a}$. We call a morphism between two quintets an isomorphism if it is invertible and the inverse is also a morphism. The set of isomorphism classes of quintets of index $\mu$ and level $\nu$ is denoted by $\mathcal{M}(\mu, \nu)$. By Theorem 4.6, we obtain a natural bijection

$$
\chi: \mathcal{M}(\mu, \nu) \longrightarrow \mathcal{S}(\mu, \nu) / \Gamma_{a}
$$




\section{Classification of commuting ordinary differential operators.}

In this section, we prove that the set $\mathcal{M}(0,-1)$ of isomorphism classes of quintets which has been introduced in Section 4 gives a complete classification of commutative algebras of ordinary differential operators containing a monic element.

We start this section with defining an extended differential algebra $\widetilde{R}$ of $R=$ $k[[x]]$.

Definition 5.1. We denote by $\widetilde{R}$ a commutative differential algebra defined over a field $k$ of characteristic zero satisfying the following conditions:

(1) $R \subset \widetilde{R}$,

(2) $k=\left\{f \in \widetilde{R} \mid \frac{d f}{d x}=0\right\}$, where $\frac{d}{d x}$ is the derivation operator.

Two important examples of such extension are $k((x))$ and $k((x))[\log x]$. We denote by $\widetilde{D}$ the ring of all ordinary differential operators with coefficients in $\widetilde{R}$. Following J.-L. Verdier [V], we use the terminology of ellipticity as follows:

Definition 5.2. A commutative $k$-subalgebra $B$ of $\widetilde{D}$ is said to be elliptic if $B$ has a monic element $P$ of order greater than zero such that every coefficient of $P$ belongs to $R$.

It is interesting to know that the commutativity of $B$ forces all the elements of $B$ to have regular coefficients.

Lemma 5.3. Every elliptic commutative subalgebra $B \subset \widetilde{D}$ is indeed a subalgebra of $D$, where $D$ denotes the ring of all differential operators with coefficients in $R$.

Proof. Let $P \in B$ be the regular monic element of order $N>0$ of Definition 5.2. Because of Lemma 7.5, there is an invertible regular pseudo-differential operator $X \in E$ of order zero such that $\partial^{N}=X^{-1} P X$. Define

$$
A=X^{-1} B X
$$

Since $B$ is commutative, we have

$$
\begin{aligned}
0 & =X^{-1}[Q, P] X \\
& =\left[X^{-1} Q X, X^{-1} P X\right] \\
& =\left[X^{-1} Q X, \partial^{N}\right]
\end{aligned}
$$

for every $Q \in B$. It is easy to check that if a pseudo-differential operator with coefficients in $\widetilde{R}$ commutes with $\partial^{N}$, then every coefficient of it is a constant. Therefore, $X^{-1} Q X \in k\left(\left(\partial^{-1}\right)\right)$, namely, $A \subset k\left(\left(\partial^{-1}\right)\right)$. This means that

$$
B=X A X^{-1} \subset X k\left(\left(\partial^{-1}\right)\right) X^{-1} \cap \widetilde{D} \subset E \cap \widetilde{D}=D .
$$


This completes the proof.

For an elliptic commutative subalgebra $B \subset D$, we define

$$
\operatorname{rank} B=\text { G.C.D. }\{\operatorname{ord} Q \mid Q \in B\}
$$

We denote by $\mathcal{B}_{r}$ the set of all elliptic commutative subalgebras of $D$ of rank $r$.

From algebraic point of view, it is natural to identify two elements of $D$ if one is obtained from the other by an inner automorphism of $D$. But since only invertible elements of $D$ are invertible functions, the above identification means that $P \in D$ and $f P f^{-1}$ are identified for all $f \in R^{\times}$. It is also usually done in analysis.

Definition 5.4. The commutative algebras $B_{1}$ and $B_{2}$ in $\mathcal{B}_{r}$ are said to be equivalent if there is an invertible element $f \in R^{\times}$such that

$$
B_{1}=f \cdot B_{2} \cdot f^{-1}
$$

We denote by $\overline{\mathcal{B}}_{r}$ the set of equivalence classes of elliptic commutative subalgebras of $D$ of rank $r$.

We need a couple of more notations.

\section{Definition 5.5.}

(1) The set $\mathcal{S}_{r}^{+}(0,-1) / \Gamma_{a}$ consists of the isomorphism classes of Schur pairs $(A, W)$ of rank $r$, index 0 and level -1 such that $W$ belongs to the big cell of the Grassmannian $G^{+}(0,-1)$.

(2) The set $\mathcal{M}_{r}^{+}(0,-1)$ consists of the isomorphism classes of quintets

$$
(C, p, \mathcal{F}, \pi, \phi)
$$

of rank $r$, index 0 and level -1 such that the torsion free sheaf $\mathcal{F}$ satisfies

$$
\operatorname{dim}_{k} H^{0}(C, \mathcal{F})=\operatorname{dim}_{k} H^{1}(C, \mathcal{F})=0 .
$$

Because of Theorem 2.7, the bijection (4.9) induces a natural bijection between $\mathcal{S}_{r}^{+}(0,-1) / \Gamma_{a}$ and $\mathcal{M}_{r}^{+}(0,-1)$.

Theorem 5.6. There is a canonical bijection

$$
\mu_{r}: \overline{\mathcal{B}}_{r} \longrightarrow \mathcal{M}_{r}^{+}(0,-1)
$$

for every $r \geq 1$. Moreover, because of Proposition 3.8, every vector bundle $\mathcal{F}$ corresponding to a commutative algebra of ordinary differential operators is semistable. 
Proof. In order to prove the theorem, it suffices to construct a canonical bijection

$$
\beta_{r}: \overline{\mathcal{B}}_{r} \longrightarrow \mathcal{S}_{r}^{+}(0,-1) / \Gamma_{a},
$$

because we have the natural bijection $\chi: \mathcal{M}_{r}^{+}(0,-1) \longrightarrow \mathcal{S}_{r}^{+}(0,-1) / \Gamma_{a}$.

Let $[B] \in \overline{\mathcal{B}}_{r}$ be the equivalence class of an elliptic commutative subalgebra $B$ of rank $r$. Choose an arbitrary element $P$ of $B$ of order greater than zero. Then there is an invertible operator $X \in E^{(0)}$ of order zero such that $X^{-1} P X \in k\left(\left(\partial^{-1}\right)\right)$ (Lemma 7.5). Since every element of $X^{-1} B X$ commutes with an operator $X^{-1} P X$ which has only constant coefficients, we know that $X^{-1} B X \subset k\left(\left(\partial^{-1}\right)\right)$. Now define $A=X^{-1} B X$ and $W=X^{-1} \rho(D) \in G^{+}(0,-1)$. By Sato's lemma (Lemma 7.2), we know that $\rho(D)=V_{0}$ is a $B$-module. Therefore,

$$
\begin{aligned}
A W & =X^{-1} B X \cdot X^{-1} V_{0} \\
& =X^{-1} B V_{0} \\
& \subset X^{-1} V_{0} \\
& =W .
\end{aligned}
$$

This means that $W$ is an $A$-module. Of course, we have

$$
\begin{aligned}
r=\operatorname{rank} B & =\text { G.C.D. }\{\operatorname{ord} Q \mid Q \in B\} \\
& =\text { G.C.D. }\left\{\operatorname{ord}\left(X^{-1} Q X\right) \mid Q \in B\right\} \\
& =\operatorname{rank} A .
\end{aligned}
$$

Thus we have constructed a Schur pair $(A, W) \in \mathcal{S}_{r}^{+}(0,-1)$. If we have another invertible operator $X_{1} \in E^{(0)}$ such that $X_{1}^{-1} B X_{1} \subset k\left(\left(\partial^{-1}\right)\right)$, then $T=X^{-1} X_{1}$ is an admissible operator. Define $A_{1}=X_{1}^{-1} B X_{1}$ and $W_{1}=X_{1}^{-1} V_{0}$. Then we have

$$
A=X^{-1} B X=X^{-1} X_{1} A X_{1}^{-1} X=T A_{1} T^{-1}
$$

and

$$
W=X^{-1} V_{0}=X^{-1} X_{1} W_{1}=T W_{1} .
$$

Therefore, $(A, W)$ and $\left(A_{1}, W_{1}\right)$ belong to the same isomorphism class of

$$
\mathcal{S}_{r}^{+}(0,-1) / \Gamma_{a} .
$$

Let $f \in R^{+}$be an invertible function. By using $f X$ instead of $X$ in the above construction, we end up with the same Schur pair

$$
(A, W)=\left((f X)^{-1} f B f^{-1}(f X),(f X)^{-1} V_{0}\right)
$$

because of the fact $f V_{0}=V_{0}$. 
Thus we have constructed a well-defined map

$$
\beta_{r}: \overline{\mathcal{B}}_{r} \longrightarrow \mathcal{S}_{r}^{+}(0,-1) / \Gamma_{a} .
$$

In order to show the bijectivity of this map, let us construct its inverse. So we start with a Schur pair $(A, W) \in \mathcal{S}_{r}^{+}(0,-1)$. Since $W \in G^{+}(0,-1)$, it defines a unique operator $S \in \Gamma_{m}$ by Sato's theorem (Theorem 7.4). This operator satisfies that $S W=V_{0}$. Now let us define

$$
B=S A S^{-1} \subset E,
$$

where we regard $A$ as a subspace of $k\left(\left(\partial^{-1}\right)\right)$. We need to show that $B=S A S^{-1} \subset$ $D$. But since $A W \subset W$ by definition, we have

$$
\begin{aligned}
B \cdot V_{0} & =S A S^{-1} \cdot S W \\
& =S A W \\
& \subset S W \\
& =V_{0} .
\end{aligned}
$$

Hence again by Sato's lemma, we conclude that $B \subset D$. Thus every Schur pair $(A, W)$ in $\mathcal{S}_{r}^{+}(0,-1)$ gives rise to an elliptic commutative algebra of ordinary differential operators.

Let us take another Schur pair $\left(A_{1}, W_{1}\right)$ such that $\left(A_{1}, W_{1}\right)=T \cdot(A, W)$ for some $T \in \Gamma_{a}$. The point $W_{1}=T W$ of the Grassmannian determines another monic integral operator $S_{1}$ so that $W_{1}=S_{1}^{-1} V_{0}$. Therefore,

$$
S_{1}^{-1} V_{0}=W_{1}=T W=T S^{-1} V_{0},
$$

i.e. $S_{1} T S^{-1} V_{0}=V_{0}$, which implies that $S_{1} T S^{-1} \in D$. But since $S_{1} T S^{-1}$ is a pseudo-differential operator of order zero, $f=S_{1} T S^{-1}$ is indeed an invertible function in $R^{\times}$. By the same argument that we used in the above, we know that $B_{1}=S_{1} A_{1} S_{1}^{-1} \subset D$. Now, we have

$$
\begin{aligned}
B_{1} & =S_{1} A_{1} S_{1}^{-1} \\
& =f \cdot S \cdot T^{-1} A_{1} T \cdot S^{-1} \cdot f^{-1} \\
& =f \cdot S \cdot T^{-1} \cdot T A T^{-1} \cdot T \cdot S^{-1} \cdot f^{-1} \\
& =f \cdot S \cdot A \cdot S^{-1} \cdot f^{-1} \\
& =f \cdot B \cdot f^{-1} .
\end{aligned}
$$

Therefore, $B_{1}$ and $B$ are equivalent. Thus we have established that the map $\beta_{r}$ is bijective. This completes the proof. 
Remark. The equivalence relation in $\mathcal{B}_{r}$ defined by the action of an invertible function does not correspond to a morphism of $\overline{\mathcal{S}}(\nu)$.

Let $\mathcal{B}_{\max }$ be the set of all maximal elliptic commutative subalgebras of $D$. Then for every $B \in \mathcal{B}_{\max }$ of rank $r$, the bijection $\beta_{r}$ gives a maximal Schur pair $\left(A_{W}, W\right)$, where $A_{W}=\{v \in V \mid v W \subset W\}$. On the other hand, since the Schur map

$$
s: G_{\text {fin }}(0,-1) \longrightarrow \bigcup_{r \in \mathbb{N}} \mathcal{S}_{r}(0,-1)
$$

of (1.7) is injective, a maximal Schur pairs can be identified with a point of the Grassmannian itself by

$$
W \longleftrightarrow\left(A_{W}, W\right)
$$

Therefore, we obtain the following.

Corollary 5.7. Let $\overline{\mathcal{B}}_{\max }$ denote the subset of $\bigcup_{r \in \mathbb{N}} \overline{\mathcal{B}}_{r}$ consisting of equivalence classes of maximal commutative algebras and let

$$
\begin{aligned}
& G_{\text {fin }}^{+}(0,-1) \\
= & \left\{W \in G(0,-1) \mid A_{W} \neq k \text { and } \operatorname{Ker} \alpha(-1)_{W}=\operatorname{Coker} \alpha(-1)_{W}=0\right\} .
\end{aligned}
$$

Then there is a natural bijection

$$
\beta: \overline{\mathcal{B}}_{\max } \longrightarrow G_{\text {fin }}^{+}(0,-1) / \Gamma_{a} .
$$

Let us consider the rank one case. From Definition 2.4, we see that the set $\mathcal{M}_{1}^{+}(0,-1)$ consists of $(C, p, v, \mathcal{F})$, where $C$ and $p$ are as before, $\mathcal{F}$ is a torsion free rank one sheaf with vanising cohomologies, and $v \in T_{p} C$ is a non-zero tangent vector of the curve $C$ at the non-singular point $p$. The morphism of (2.7) absorbs all information of $\phi$ and hence we do not have it any more in the isomorphism class of $\mathcal{M}_{1}^{+}(0,-1)$. But since we restricted the shape of the isomorphism $\bar{h}$ as in (2.5), it does not absorb $\pi$ entirely. Indeed, the leading coefficient of $\pi$ is invariant under the application of $\bar{h}$, and which can be identified naturally as a non-zero tangent vector. Therefore, in the case of rank one, our Theorem 5.6 recovers exactly the Krichever's theorem in its precise form of statement which was given by Mumford [Mum].

\section{The KP flows on the quotients of the Grassmannians.}

Throughout this section, we assume that $k$ is a field of characteristic zero. In this section, we define the KP flows on certain quotient spaces of the Grassmannians. The result of [M1] and [M2] shows that every finite dimensional orbit of these flows has a structure of the Jacobian variety of an algebraic curve. Combining this result 
with the theorem of categorical equivalence (Theorem 3.7) and the recent theory of Hitchin $[\mathrm{H}]$ and Beauville-Narasimhan-Ramanan [BNR], one can prove that the $\mathrm{KP}$ flows produce all generic vector bundles on an arbitrary algebraic curve of genus greater than one.

Let us begin with defining the quotient space of $G(\mu, \nu)$. Let

$$
\Gamma_{c}=1+k\left[\left[\partial^{-1}\right]\right] \cdot \partial^{-1}=1+V^{(-1)}
$$

be the group of monic pseude-differential operators with constant coefficients. Because of the fact that $A_{W} \cap \Gamma_{c}=\{1\}$ by (3.1), the action of $\Gamma_{c}$ on $G(\mu, \nu)$ is fixed-point-free. Hence we can define the quotient space

$$
X(\mu, \nu)=G(\mu, \nu) / \Gamma_{c}
$$

and we denote the natural projection by

$$
\Pi: G(\mu, \nu) \longrightarrow X(\mu, \nu)
$$

Our Grassmannian $G(\mu, \nu)$ has a structure of the principal $\Gamma_{c}$-bundle over $X(\mu, \nu)$.

Every element $P \in E$ defines a vector field $\Phi(P)$ on each $G(\mu, \nu)$ by (4.7). We call a vector field

$$
\varepsilon: G(\mu, \nu) \ni W \longmapsto \varepsilon_{W} \in \operatorname{Hom}(W, V / W)
$$

left $\Gamma_{c}$-invariant if

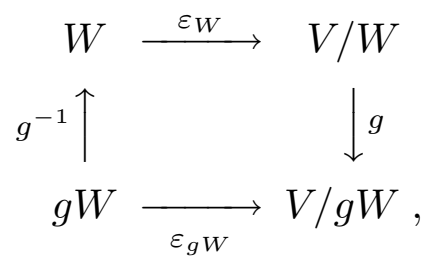

i.e. $\varepsilon_{g W}=g \cdot \varepsilon_{W} \cdot g^{-1}$ for every $g \in \Gamma_{c}$. Note that since $\Gamma_{c}$ action has no fixed points, $g \in \Gamma_{c}$ defines canonical maps

$$
g W \ni g w \stackrel{g^{-1}}{\longmapsto} w \in W
$$

and

$$
V / W \ni v \bmod W \stackrel{g}{\longmapsto} g v \bmod g W \in V / g W .
$$

A vector field on $G(\mu, \nu)$ does not descend to $X(\mu, \nu)$ in general, but left $\Gamma_{c}$-invariant ones do. For every operator $P \in E, \Phi\left(\rho\left(P_{-}\right)\right)$gives the vertical component of the vector field $\Phi(P)$ because $\rho\left(P_{-}\right)$is an element of the Lie algebra $V^{(-1)}$ of the commutative group $\Gamma_{c}$. In this sense, $\rho\left(\bullet_{-}\right)$can be viewed as a connection of the principal $\Gamma_{c}$-bundle. For arbitrary two points $W$ and $W^{\prime}$ of a fiber of $\Pi$, there 
is a unique element $g \in \Gamma_{c}$ such that $W^{\prime}=g W$, and which induces a canonical isomorphism

$$
\operatorname{Hom}(W, V / W) / \Phi_{W}\left(V^{(-1)}\right) \cong \operatorname{Hom}(g W, V / g W) / \Phi_{g W}\left(V^{(-1)}\right) .
$$

Therefore, we can define the tangent space of $X(\mu, \nu)$ at a point $\Pi(W)$ by

$$
T_{\Pi(W)} X(\mu, \nu)=\operatorname{Hom}(W, V / W) / \Phi_{W}\left(V^{(-1)}\right),
$$

because the right hand side does not depend on the choice of the point $W \in$ $\Pi^{-1}(\Pi(W))$.

Since every $v \in V$ commutes with $\Gamma_{c}$ and we have

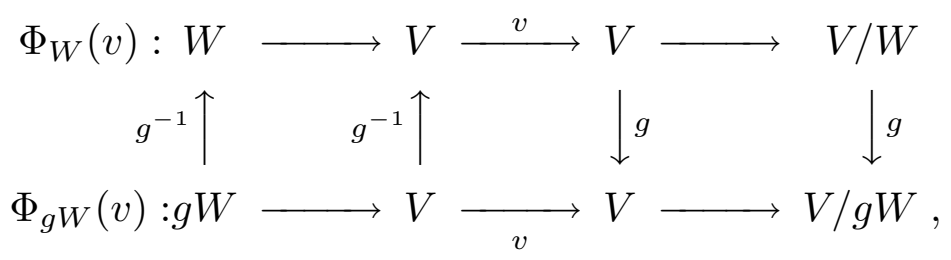

the vector field $\Phi(v)$ on a Grassmannian defines a vector field

$$
\Pi(W) \longmapsto \hat{\Phi}_{\Pi(W)}(v)=\Phi_{W}(v) \bmod \Phi_{W}\left(V^{(-1)}\right)
$$

on the quotient space $X(\mu, \nu)$, and the definition does not depend on the choice of a point on the fiber. Thus we obtain the set $\hat{\Phi}(V)$ of infinitely many commuting vector fields on the quotient space. In this case, we also say that $V$ has an infinitesimal action on the quotient space $X(\mu, \nu)$.

Definition 6.1. The infinite set $\hat{\Phi}(V)$ of commuting vector fields on $X(\mu, \nu)$ is called the (generalized) KP flows.

Remark. The infinitesimal action of $t_{n} z^{-n} \in V$ on a point $\Pi(W) \in G^{+}(0,-1) / \Gamma_{c}$ corresponds to the $n$-th Lax equation of the KP system

$$
\frac{\partial L}{\partial t_{n}}=\left[\left(L^{n}\right)_{+}, L\right]
$$

of [M2], where $L=S \cdot \partial \cdot S^{-1}$ is a monic operator of order one corresponding to $\Pi(W)$ (which is called the Lax operator) and $S$ is the monic operator of order zero corresponding to $W$ by the theorem of Sato (Theorem 7.4). Note that the ambiguity of determining $S$ from $L$ coincides exactly with the $\Gamma_{c}$-action on the big cell $G^{+}(0,-1)$. 
Definition 6.2. An integral manifold of the KP flows is a subvariety $M \subset X(\mu, \nu)$ such that $T_{\Pi(W)} M$ coincides with $\hat{\Phi}_{\Pi(W)}(V)$ as a subspace of the tangent space $T_{\Pi(W)} X(\mu, \nu)$ for every $\Pi(W) \in M$.

Theorem 6.3. (cf. [M1], [M2]) Every finite dimensional integral manifold of the $K P$ flows on $X(\mu, \nu)$ has a natural structure of the (generalized) Jacobian variety of an algebraic curve. Moreover, the KP flows restricted on an integral manifold is linear with respect to the natural linear structure of the Jacobian variety.

Proof. Let $M$ be a finite dimensional integral manifold of the KP flows and let $\Pi(W)$ be a point of $M$, where $W \in G(\mu, \nu)$. The maximal stabilizer of $W$ is given by $A_{W}=\{v \in V \mid v W \subset W\}$.

Consider the linear map $\hat{\Phi}_{\Pi(W)}: V \longrightarrow \operatorname{Hom}(W, V / W) / \Phi_{W}\left(V^{(-1)}\right)$. Since $\operatorname{Ker} \hat{\Phi}_{\Pi(W)}=A_{W}+V^{(-1)}$, we have

$$
T_{\Pi(W)} M \cong V /\left(A_{W}+k[[z]]\right) .
$$

The finite dimensionality of $M$ thus implies that rank $A_{W}=1$. Therefore, we obtain a rank one Schur pair $\left(A_{W}, W\right)$, which corresponds to a quintet $(C, p, \mathcal{L}, \pi, \phi)$ with a line bundle $\mathcal{L}$. If we use $T W$ instead of $W$, where $T \in \Gamma_{c}$, then we obtain another Schur pair $\left(T A_{W} T^{-1}, T W\right)=\left(A_{W}, T W\right)$ and the corresponding quintet $\left(C^{\prime}, p^{\prime}, \mathcal{L}^{\prime}, \pi^{\prime}, \phi^{\prime}\right)$. These two quintets are isomorphic to one another in the sense of Definition 2.4. Note that the $\Gamma_{c}$-action on the quintets corresponds to a morphism such that the isomorphism $h$ of (2.5) and (2.6) is the identity. In other words, every point $\Pi(W)$ of $M$ corresponds to an isomorphism class $(C, P, \mathcal{L}, \pi) \in \mathcal{Q}_{1}(\mu, \nu) / \Gamma_{c}$, because (2.7) wipes out the local trivialization $\phi$ completely when $\operatorname{rank} \mathcal{L}=1$. Under this correspondence, we have the canonical isomorphism

$$
T_{\Pi(W)} M \cong V / \operatorname{Ker} \hat{\Phi}_{\Pi(W)} \cong H^{1}\left(C, \mathcal{O}_{C}\right)
$$

which follows from the argument of the proof of Theorem 3.3. This isomorphism means that the vector fields in $\hat{\Phi}(V)$ produce infinitesimal deformations of the line bundle $\mathcal{L}$. Therefore, integration of these vector fields must give the real deformations of $\mathcal{L}$.

Every element $v=t_{1} z^{-1}+t_{2} z^{-2}+\cdots+t_{n} z^{-n} \in V$, where $n$ is a sufficiently large integer, defines a cohomology element $\bar{v} \in H^{1}\left(C, \mathcal{O}_{C}\right)$ by the above isomorphism. In order to integrate the vector field $\hat{\Phi}(v)$, we need the exponential function. Here, we have the natural exponential map exp : $H^{1}\left(C, \mathcal{O}_{C}\right) \longrightarrow \operatorname{Pic}^{0}(C)$. So let $\ell(\bar{v}) \in \operatorname{Pic}^{0}(C)$ be the line bundle over $C$ corresponding to $\bar{v}=\exp \left(t_{1} z^{-1}+t_{2} z^{-2}+\right.$ $\left.\cdots+t_{n} z^{-n}\right)$. (Note that after replacing $z$ by $\partial^{-1}$, this quantity becomes the time evolution operator of the KP system which was studied in [M3].) Now we identify

$$
\operatorname{Pic}^{d}(C)=\left\{(C, p, \pi, \mathcal{L} \otimes \ell(\bar{v})) \mid \ell(\bar{v}) \in P i c^{0}(C)\right\},
$$


where $d=\operatorname{deg} \mathcal{L}=\mu+\operatorname{genus}(C)-1$. Since $\ell(\bar{v})$ gives the real deformation of $\mathcal{L}$ by $\mathcal{L} \otimes \ell(\bar{v})$, we can conclude that $M \cong \operatorname{Pic}^{d}(C)$. The KP flows are nothing but the infinitesimal version of this deformation, hence they are linear. If we regard $\operatorname{Pic}^{d}(C)$ as the Jacobian variety of $C$, then we have completed the proof.

Since the infinitesimal $V$-action on the Grassmannians does not affect on the stabilizers $A, V$ defines an infinitesimal action on the set of Schur pairs $\mathcal{S}_{r}(\mu, \nu)$ of arbitrary rank $r$, and hence the the set $\mathcal{S}_{r}(\mu, \nu) / \Gamma_{c}$ of isomorphism classes. Therefore, we have an infinitesimal $V$-action on the set $\mathcal{Q}_{r}(\mu . \nu) / \Gamma_{c}$ of isomorphism classes of the higher rank quintets.

Let $\mathcal{F}$ be a generic semistable vector bundle of degree $d$ and rank $r$ defined on a smooth algebraic curve $C$ of genus $g>1$. It is shown in [BNR] that there is an $r$-sheeted ramified covering $\beta: \widetilde{C} \longrightarrow C$ over $C$ and a line bundle $\mathcal{L}$ defined over $\widetilde{C}$ such that $\mathcal{F} \cong \beta_{*} \mathcal{L}$.

Choose a ramification point $p \in C$ of $\beta$, a point $\tilde{p} \in \beta^{-1}(p)$, a formal scheme isomorphism $\pi: U_{0} \longrightarrow U_{\tilde{p}}$ and an $\mathcal{O}_{U_{\tilde{p}}}$-module isomorphism $\phi: \mathcal{L}_{U_{\tilde{p}}} \longrightarrow \pi_{*} \mathcal{O}_{U_{0}}(\nu)$, as in Definition 2.1, where $\nu$ is an arbitrarily fixed integer. Define $\mu=d-r(g-1)$. Thus we have constructed a quintet $(\tilde{C}, \tilde{p}, \mathcal{L}, \pi, \phi)$ of index $\mu$, level $\nu$ and rank 1 . It corresponds to a Schur pair $(\tilde{A}, \tilde{W})$.

But we have another quintet $\left(C, p, \mathcal{F}, \widehat{\beta} \circ \pi, \pi_{*}(\phi)\right)$ of the same index $\mu$ and level $\nu$ but of rank $r$, which gives a Schur pair $(A, W)$. Let us choose an isomorphism $\psi: \mathcal{F} \longrightarrow \beta_{*} \mathcal{L}$. Then we have a morphism

$$
(\beta, \psi):(\tilde{C}, \tilde{p}, \mathcal{L}, \pi, \phi) \longrightarrow\left(C, p, \mathcal{F}, \widehat{\beta} \circ \pi, \pi_{*}(\phi)\right)
$$

which corresponds to an admissible operator $T \in \Gamma_{a}$. Since $\beta$ is a projection and $\psi$ is an isomorphism, $T$ gives $T A T^{-1}=A \subset \tilde{A}$ and $T W=\tilde{W}$. Therefore, $T$ is actually an element of $\Gamma_{c}$ and $\Pi(W)$ determines a unique point of the space $X(\mu, \nu)$. Now consider the $V$-action (i.e. the integrated $\mathrm{KP}$ flows) on $X(\mu, \nu)$ at $\Pi(W)$. On the set $\mathcal{Q}_{1}(\mu, \nu) / \Gamma_{c}$ in which $(\tilde{C}, \tilde{p}, \mathcal{L}, \pi, \phi)$ belongs, the $V$-action produces $\operatorname{Pic}^{\delta}(\widetilde{C})$, where $\delta=\operatorname{deg} \mathcal{L}$. Since each point $\left(\tilde{C}, \tilde{p}, \mathcal{L} \otimes \ell(\bar{v}), \pi, \phi_{\bar{v}}\right)$ of the orbit defines a point $\left(C, p, \beta_{*}(\mathcal{L} \otimes \ell(\bar{v})), \widehat{\beta} \circ \pi, \pi_{*}\left(\phi_{\bar{v}}\right)\right)$ of $\mathcal{Q}_{r}(\mu, \nu) / \Gamma_{c}, V$ acts on $\mathcal{Q}_{r}(\mu, \nu) / \Gamma_{c}$ at the point $\left(C, p, \mathcal{F}, \widehat{\beta} \circ \pi, \pi_{*}(\phi)\right)$. In other words, we have introduced a $V$ action on the generic points of $\mathcal{Q}_{r}(\mu, \nu) / \Gamma_{c}$. According to the result of [BNR], the generic vector bundles on $C$ can be constructed as $\beta_{*}(\mathcal{L} \otimes \ell(\bar{v}))$. Thus we have established the following.

Theorem 6.4. Let $(C, p, \mathcal{F}, \pi, \phi) \in \mathcal{Q}_{r}(\mu, \nu) / \Gamma_{c}$ be an isomorphism class of a quintet consisting of a smooth curve $C$ of genus greater than one and a generic semistable vector bundle $\mathcal{F}$ of arbitrary rank and degree. We can define the KP action on this quintet through the KP flows on $X(\mu, \nu)$ at $\Pi(W)$, where $(A, W)$ is the Schur pair corresponding to the quintet. Then the orbit of the KP action on $\mathcal{Q}_{r}(\mu, \nu) / \Gamma_{c}$ is 
finite dimensional and contains all generic vector bundles on $C$ of the given degree and rank.

\section{Appendix - a theorem of M. Sato.}

In this section, we give a proof of the theorem of Sato [S], [SN]. The theorem is stated in a little different formulation from the original one in order to make the correspondence canonical. We also give a proof of the interesting characterization of differential operators among pseudo-differential operators due to him. This characterization corresponds to our stabilizer condition $A W \subset W$, and hence it gives the origin of the reason why a commutative algebra of ordinary differential operators produces not only an algebraic curve but also a vector bundle on it.

Let $k$ be a field of characteristic zero. In Section 4 , we introduced our function space $R=k[[x]]$. In this section, we need a topology of $R$. So let $m_{x} \subset R$ be the unique maximal ideal. Since

$$
\bigcup_{n=0}^{\infty} m_{x}^{n}=R \text { and } \bigcap_{n=0}^{\infty} m_{x}^{n}=\{0\}
$$

$R$ is a complete topological space with respect to the $m_{x}$-adic topology.

Definition 7.1. We define the valuation $\operatorname{val}_{x}(f)$ of an element $f \in R$ by

$$
\begin{aligned}
\operatorname{val}_{x}(f)=n & \Longleftrightarrow f \in m_{x}^{n} \backslash m_{x}^{n+1} \\
& \Longleftrightarrow f \text { has a zero of order } n \text { at } x=0 .
\end{aligned}
$$

Let $E$ be the ring of pseudo-differential operators and let $D$ be the subring of differential operators. We have a natural direct sum decomposition

$$
E=D \oplus E^{(-1)}
$$

as a left, right or both-sided $R$-module. According to this decomposition, we write $P=P_{+}+P_{-}$, where $P_{+} \in D$ and $P_{-} \in E^{(-1)}$. For an operator

$$
P=\sum_{n \in \mathbb{Z}} f_{n} \partial^{n}=\sum_{m \in \mathbb{Z}} \partial^{m} \cdot g_{m} \in E
$$

we have

$$
\begin{aligned}
& P_{+}=\sum_{n \geq 0} f_{n} \partial^{n}=\sum_{m \geq 0} \partial^{m} \cdot g_{m} \quad \text { and } \\
& P_{-}=\sum_{n<0} f_{n} \partial^{n}=\sum_{m<0} \partial^{m} \cdot g_{m}
\end{aligned}
$$


Thus the decomposition (7.1) does not depend on the expression of the operator.

Let us recall the natural projection $\rho: E \longrightarrow E / E x$. Obviously, we have $\rho(D)=$ $k\left[z^{-1}\right], \rho\left(E^{(-1)}\right)=V^{(-1)}$ and hence $V=k\left[z^{-1}\right] \oplus V^{(-1)}$, which corresponds to the natural direct sum decomposition $E=D \oplus E^{(-1)}$.

The following lemma due to M. Sato [SN] gives an intersting characterization of differential operators among pseudo-differential operators and plays a central role in the determination of commutative algebras of ordinary differential operators in Section 5.

Lemma 7.2. A pseudo-differential operator $P \in E$ is a differential operator if and only if it preserves $\rho(D)$ in $V$, namely

$$
P \rho(D) \subset \rho(D)
$$

Proof. Every differential operator $P \in D$ preserves $\rho(D)$ because $P \rho(Q)=\rho(P Q) \in$ $\rho(D)$ for any $Q \in D$. In order to prove the converse, we need the $m_{x}$-adic topology of the function space $R$. Let $P$ be a pseudo-differential operator and let

$$
P_{-}=\sum_{n=1}^{\infty} \partial^{-n} \cdot f_{n}(x)
$$

be the $E^{(-1)}$-part of $P$ according to the decomposition of (7.1). The condition $P \rho(D) \subset \rho(D)$ implies that $P D \bmod E x \subset D \bmod E x$, that is

$$
(P Q)_{-} \in E x
$$

for every $Q \in D$. Therefore, $P_{-} \in E x$ because $P \cdot 1 \bmod E x \in D \bmod E x$. Thus $\operatorname{val}_{x} f_{n} \geq 1$ for all $n \geq 1$. So let $f_{m}$ be the coefficient of $(7.2)$ with the lowest valuation and let $\operatorname{val}_{x} f_{m}=\ell \geq 1$. Consider the operator $\left(P \cdot \partial^{\ell}\right)_{-}$. Then we have

$$
\begin{aligned}
\left(P \cdot \partial^{\ell}\right)_{-} & =\left(P_{-} \cdot \partial^{\ell}\right)_{-} \\
& =\left(\sum_{n=1}^{\infty} \partial^{-n} \cdot f_{n} \cdot \partial^{\ell}\right)_{-} \\
& =\left(\sum_{n=1}^{\infty} \sum_{i=0}^{\ell} \partial^{-n+\ell-i}(-1)^{i}\left(\begin{array}{l}
\ell \\
i
\end{array}\right) f_{n}^{(i)}\right)_{-} \\
& =\left(\sum_{j=1}^{\infty} \partial^{\ell-j} \sum_{i=0}^{\ell}(-1)^{i}\left(\begin{array}{l}
\ell \\
i
\end{array}\right) f_{j-i}^{(i)}\right)_{-} \\
& =\sum_{j=\ell+1}^{\infty} \partial^{\ell-j} \sum_{i=0}^{\ell}(-1)^{i}\left(\begin{array}{l}
\ell \\
i
\end{array}\right) f_{j-i}^{(i)} \cdot
\end{aligned}
$$


Since $f_{n}^{(i)}(0)=0$ for $0 \leq i<\ell$, we have

$$
\begin{aligned}
\rho\left(\left(P \cdot \partial^{\ell}\right)_{-}\right) & =\sum_{j=\ell+1}^{\infty}(-1)^{\ell} f_{j-\ell}^{(\ell)}(0) \cdot \partial^{\ell-j} \\
& =\sum_{j=1}^{\infty}(-1)^{\ell} f_{j}^{(\ell)}(0) \partial^{-j} .
\end{aligned}
$$

But we know that $\left(P \cdot \partial^{\ell}\right)_{-} \in E x$ by (7.3). Thus $f_{n}^{(\ell)}(0)=0$ for all $n \geq 1$. This means that $\operatorname{val}_{x} f_{m}>\ell$. But this contradicts with our assumption. Therefore, none of the coefficient $f_{n}$ can have the lowest valuation. Namely, $f_{n}(x)=0$ for all $n \geq 1$. This means that $P$ is a differential operator. This completes the proof.

Let us define another Grassmannian consisting of right $D$-modules.

Definition 7.3. The Sato Grassmannian is the set

$$
S G^{+}=\left\{J \subset E \text { closed subspace of } E \mid J \oplus E^{(-1)}=E \text { and } J D \subset J\right\}
$$

consisting of the right D-modules which are direct summand of $E$.

The following theorem is also due to Sato (cf. [SN] ).

\section{Theorem 7.4 .}

(1) Let $\Gamma_{m}$ be the group of monic zero-th order pseudo-differential operators and let $S G^{+}$be the Sato Grassmannian defined as above. Then there is a natural bijection $\sigma: \Gamma_{m} \stackrel{\sim}{\longrightarrow} S G^{+}$given by

$$
\Gamma_{m} \ni S \stackrel{\sigma}{\longmapsto} \sigma(S)=J=S^{-1} D \in S G^{+}
$$

(2) Let $G^{+}(0,-1)$ be the big-cell of the Grassmannian of index 0 and level -1 . Then the natural projection $\rho: E \rightarrow V$ induces a bijection

$$
\rho: S G^{+} \stackrel{\sim}{\longrightarrow} G^{+}(0,-1)
$$

Proof. (1) Let $S \in \Gamma_{m}$. Obviously, $S^{-1} D=J$ is a right $D$-module. Since $S^{-1} E=$ $E$ and $S^{-1} E^{(-1)}=E^{(-1)}$, we have

$$
\begin{aligned}
E & =S^{-1} E=S^{-1}\left(D \oplus E^{(-1)}\right)=S^{-1} D \oplus S^{-1} E^{(-1)} \\
& =S^{-1} D \oplus E^{(-1)} .
\end{aligned}
$$

Thus $S^{-1} D \in S G^{+}$. 
Injectivity of $\sigma$.

Suppose that $\sigma\left(S_{1}\right)=\sigma\left(S_{2}\right)=J$. Then $S_{1}^{-1} D=S_{2}^{-1} D$, which means $S_{1} S_{2}^{-1} D=$ $D$. For the operator $1 \in D$, it gives $S_{1} S_{2}^{-1} \cdot 1=S_{1} S_{2}^{-1} \in D$. But since $S_{1} \cdot S_{2}^{-1} \in \Gamma_{m}$ and $\Gamma_{m} \cap D=\{1\}$, we have $S_{1} \cdot S_{2}^{-1}=1$ i.e. $S_{1}=S_{2}$.

Subjectivity of $\sigma$.

Let $J \in S G^{+}$be an arbitrary element. Since $E=J \oplus E^{(-1)}, J$ contains a monic zero-th order operator. Choose $S \in \Gamma_{m}$ such that $S^{-1} \in J$ and let $S^{-1} D$ be the right $D$-module generated by $S^{-1}$ in $E$. Since $J$ is itself a right $D$-module, $S^{-1} D \subset J$.

Now let $P \in J$ be an arbitrary element of order $N \geq 0$. Since $S^{-1} \in J$ is monic of order 0 , there is a differential operator $Q_{N} \in D$ of order $N$ such that

$$
P-S^{-1} Q_{N} \in J^{(N-1)}=J \cap E^{(N-1)} .
$$

We can use the same argument to find a $Q_{N-1} \in D \cap E^{(N-1)}$ such that

$$
P-S^{-1} Q_{N}-S^{-1} Q_{N-1} \in J^{(N-2)}=J \cap E^{(N-2)} .
$$

If we continue this process $N$-times, then we end up with

$$
P-S^{-1} \sum_{n=0}^{N} Q_{N-n} \in J^{(-1)}=J \cap E^{(-1)}=\{0\} .
$$

Therefore, $P=S^{-1} \sum_{n=0}^{N} Q_{N-n} \in S^{-1} D$, i.e. $J \subset S^{-1} D$. Thus $J=S^{-1} D=\sigma(S)$.

(2) Let $J \in S G^{+}$be an arbitrary element. As we have established in the above, there is an $S \in \Gamma_{m}$ such that $J=S^{-1} D$. Now, we have

$$
\begin{aligned}
\rho(J) & =\rho\left(S^{-1} D\right) \\
& =\left\{\rho\left(S^{-1} Q\right) \mid Q \in D\right\} \\
& =S^{-1}\{\rho(Q) \mid Q \in D\} \\
& =S^{-1} \rho(D) .
\end{aligned}
$$

Since $S^{-1}: V \stackrel{\sim}{\rightarrow} V$ is a homeomorphism, we have

$$
V=S^{-1} V=S^{-1} \rho(D) \oplus V^{(-1)} .
$$

Thus $S^{-1} \rho(D) \in G^{+}(0,-1)$ and hence we have a well-defined map $\rho: S G^{+} \rightarrow$ $G^{+}(0,-1)$.

Injectivity of $\rho$. 
Suppose we have $\rho\left(S_{1}^{-1} D\right)=\rho\left(S_{2}^{-1} D\right)$. Then $S_{1}^{-1} \rho(D)=S_{2}^{-1} \rho(D)$, i.e.,

$$
S_{1} S_{2}^{-1} \rho(D)=\rho(D) \text {. }
$$

Therefore, $S_{1} S_{2}^{-1} \in D \cap \Gamma_{m}=\{1\}$, namely, $S_{1}=S_{2}$.

Surjectivity of $\rho$.

Let $W \in G^{+}(0,-1)$ be an arbitrary point. Since $V=W \oplus V^{(-1)}$, we can choose a basis $\left\{w_{n}\right\}_{n \geq 0}$ for $W$ in the following form for every $n \geq 0$ :

$$
w_{n}=z^{-n}+\sum_{\ell=1}^{\infty} a_{n \ell} z^{\ell}
$$

In order to construct an operator $S \in \Gamma_{m}$, let $S^{-1}=1+\sum_{\ell=1}^{\infty} \partial^{-\ell} \cdot s_{\ell}(x)$ and $s_{0}(x)=1$. Then the equation

$$
w_{0}=S^{-1} \cdot 1=\rho\left(S^{-1}\right)=1+\sum_{\ell=1}^{\infty} s_{\ell}(0) z^{\ell}
$$

determines all the constant terms of the coefficients as $s_{\ell}(0)=a_{0 \ell}, \ell \geq 1$.

Now let us assume that we know $s_{\ell}^{(i)}(0)$ for all $\ell \geq 1$ and $0 \leq i<n$. Note that we have

$$
\begin{aligned}
& S^{-1} \cdot z^{-n} \\
& =\rho\left(S^{-1} \cdot \partial^{n}\right) \\
& =\rho\left(\sum_{m=0}^{\infty} \partial^{-m} \cdot s_{m}(x) \cdot \partial^{n}\right) \\
& =\rho\left(\sum_{m=0}^{\infty} \sum_{i=0}^{n} \partial^{n-m-i} \cdot(-1)^{i}\left(\begin{array}{c}
n \\
i
\end{array}\right) s_{m}^{(i)}(x)\right) \\
& =\rho\left(\partial^{n}+\sum_{\ell=1}^{\infty} \partial^{n-\ell} \sum_{i=0}^{n}(-1)^{i}\left(\begin{array}{c}
n \\
i
\end{array}\right) s_{\ell-i}^{(i)}(x)\right) \\
& =\partial^{n}+\sum_{\ell=1}^{n-1} \partial^{n-\ell} \sum_{i=0}^{\ell}(-1)^{i}\left(\begin{array}{c}
n \\
i
\end{array}\right) s_{\ell-i}^{(i)}(0)+\sum_{i=0}^{n-1}(-1)^{i}\left(\begin{array}{c}
n \\
i
\end{array}\right) s_{n-i}^{(i)}(0) \\
& \quad+\sum_{\ell=n+1}^{\infty} \partial^{n-\ell} \sum_{i=0}^{n}(-1)^{i}\left(\begin{array}{c}
n \\
i
\end{array}\right) s_{\ell-i}^{(i)}(0) \\
& =z^{-n}+\sum_{\ell=1}^{n-1} \sum_{i=0}^{\ell}(-1)^{i}\left(\begin{array}{c}
n \\
i
\end{array}\right) s_{\ell-i}^{(i)}(0) z^{-n+\ell}+\sum_{i=0}^{n-1}(-1)^{i}\left(\begin{array}{c}
n \\
i
\end{array}\right) s_{n-i}^{(i)}(0) \\
& \quad+\sum_{\ell=1}^{\infty} \sum_{i=0}^{n}(-1)^{i}\left(\begin{array}{c}
n \\
i
\end{array}\right) s_{n+\ell-i}^{(i)}(0) z^{\ell} .
\end{aligned}
$$


The non-negative order terms of the above expression exactly coincides with

$$
w_{n}+\sum_{\ell=1}^{n-1} \sum_{i=0}^{\ell}(-1)^{i}\left(\begin{array}{c}
n \\
i
\end{array}\right) s_{\ell-i}^{(i)}(0) w_{n-\ell}+\sum_{i=0}^{n-1}(-1)^{i}\left(\begin{array}{c}
n \\
i
\end{array}\right) s_{n-i}^{(i)}(0) w_{0}
$$

which contains only known quantities. Therefore, the equation

$$
\begin{aligned}
& S^{-1} \cdot z^{-n} \\
& =z^{-n}+\sum_{\ell=1}^{n-1} \sum_{i=0}^{\ell}(-1)^{i}\left(\begin{array}{c}
n \\
i
\end{array}\right) s_{\ell-i}^{(i)}(0) z^{-n+\ell}+\sum_{i=0}^{n-1}(-1)^{i}\left(\begin{array}{c}
n \\
i
\end{array}\right) s_{n-i}^{(i)}(0) \\
& \quad+\sum_{\ell=1}^{\infty} \sum_{i=0}^{n}(-1)^{i}\left(\begin{array}{c}
n \\
i
\end{array}\right) s_{n+\ell-i}^{(i)}(0) z^{\ell} \\
& =w_{n}+\sum_{\ell=1}^{n-1} \sum_{i=0}^{\ell}(-1)^{i}\left(\begin{array}{c}
n \\
i
\end{array}\right) s_{\ell-i}^{(i)}(0) w_{n-\ell}+\sum_{i=0}^{n-1}(-1)^{i}\left(\begin{array}{c}
n \\
i
\end{array}\right) s_{n-i}^{(i)}(0) w_{0}
\end{aligned}
$$

determines $s_{\ell}^{(n)}(0)$ for all $\ell \geq 1$. Thus we have obtained $s_{\ell}(x)=\sum_{n=0}^{\infty} \frac{1}{n !} s_{\ell}^{(n)}(0) x^{n}$. Now the operator $S^{-1}=1+\sum_{\ell=1}^{\infty} \partial^{-\ell} \cdot s_{\ell}(x)$ satisfies $S^{-1} \rho(D)=W$ as required. This completes the proof.

By an easy calculation, we obtain the following.

Lemma 7.5. For every first order operator

$$
L=\partial+u_{1}+u_{2} \partial^{-1}+u_{3} \partial^{-2}+\cdots,
$$

there exists an invertible zero-th order operator

$$
X=s_{0}+s_{1} \partial^{-1}+s_{2} \partial^{-2}+\cdots
$$

such that $X^{-1} L X=\partial$. If $\bar{X}$ is another operator satisfying the same equation $\bar{X}^{-1} L \bar{X}=\partial$, then there is an invertible zero-th order operator $X_{c}$ with constant coefficients such that $\bar{X}=X \cdot X_{c}$.

\section{References}

[ADKP] E. Arbarello, C. De Concini, V. Kac and C. Procesi: Moduli spaces of curves and representation theory, Commun. Math. Phys. 117 (1988) 1-36. 
[B] J.-L. Brylinski: Loop groups and non-commutative theta functions, Penn State Preprint (1989).

[BC] J. L. Burchnall and T. W. Chaundy: Commutative ordinary differential operators, Proc. London Math. Soc. Ser. 2, 21 (1923) 420-440; Proc. Royal Soc. London Ser. A, 118 (1928) 557-583.

[BNR] A. Beauville, M. S. Narasimhan and S. Ramanan: Spectral curves and the generalized theta divisor, Journ. Reine Angew. Math. 398 (1989) $169-179$.

[BS] A. A. Beilinson and V. Schechtman: Determinant bundles and Virasoro algebra, Commun. Math. Phys. 118 (1988) 651-701.

[F] G. Floquet: Sur la théorie des équations différentielles linéaires, Ann. Sci. de l'École Norm. Supér., 8 (1879) Suppl., 3-132.

[GD] I. M. Gel'fand and L. A. Dikii: Asymptotic behaviour of the resolvent of Sturm-Liouville equations and the algebra of the Korteweg-de Vries equations, Russ. Math. Surveys, 30 (1975) 77-113; Fractional powers of operators and Hamiltonian systems, Func. Anal. Appl. 10 (1976) $259-273$.

[H] N. Hitchin: Stable bundles and integrable systems, Duke Math. J. 54 (1987) 91-114.

[K] I. M. Krichever: Methods of algebraic geometry in the theory of nonlinear equations, Russ. Math. Surveys 32 (1977) 185-214.

[KNTY] N. Kawamoto, Y. Namikawa, A. Tsuchiya and Y. Yamada: Geometric realization of conformal field theory on Riemann surfaces, Commun. Math. Phys. 116 (1988) 247-308.

[KSU] T. Katsura, Y. Shimizu and K. Ueno: Formal groups and conformal field theory over $\mathbb{Z}$, Adv. Studies in Pure Math. 19 (1989) 1001-1020.

[M1] M. Mulase: Algebraic geometry of soliton equations, Proc. Japan Acad. Ser. A, 59 (1983) 285-288.

[M2] _ Cohomological structure in soliton equations and jacobian varieties, J. Diff. Geom. 19 (1984) 403-430.

[M3] : Solvability of the super $K P$ equation and a generalization of the Birkhoff decomposition, Invent. Math. 92 (1988) 1-46.

[M4] : Geometric classification of commutative algebras of ordinary differential operators, Proc. 18-th Intern. Conf. on Differential Geometrical Methods in Theor. Phys., to appear (ITD Preprint 89/90-6). 
[Mum] D. Mumford: An algebro-geometric constructions of commuting operators and of solutions to the Toda lattice equations, Korteweg-de Vries equations and related non-linear equations, In Proc. Internat. Symp. on Alg. Geom., Kyoto 1977, Kinokuniya Publ. (1978) 115-153.

[P] S. Pincherle: Mémoire sur le calcul fonctionnel distributif, Math. Ann. 49 (1897) 325-382.

$[\mathrm{PW}] \quad$ E. Previato and G. Wilson: Vector bundles over curves and solutions of the KP equations, Proc. Symp. Pure Math. 49, AMS,(1989) 553-569.

[S] M. Sato: Soliton equations as dynamical systems on an infinite dimensional Grassmannian manifold, Kokyuroku, Res. Inst. Math. Sci., Kyoto Univ. 439 (1981) 30-46.

[SN] M. Sato and M. Noumi: Soliton equations and universal Grassmann manifold (in Japanese), Sophia Univ. Lec. Notes Ser. in Math. 18 (1984).

[Sc] I. Schur: Über vertauschbare lineare Differentialausdrücke, Sitzungsber. der Berliner Math. Gesel. 4 (1905) 2-8.

[SW] G. B. Segal and G. Wilson: Loop groups and equations of $K d V$ type, Publ. Math. I.H.E.S. 61 (1985) 5-65.

[T] K. Takasaki: Integrable systems as deformations of $D$-modules, RIMS-601 Preprint, Kyoto Univ. (1987).

[V] J.-L. Verdier: Equations differentielles algébriques, Séminaire de lÉcole Normale Supérieure 1979-82, Birkhäuser (1983) 215-236.

[W] G. Wallenberg: Über die Vertauschbarkeit homogener linearer Differentialausdrücke, Archiv der Math. u. Phys., Drittle Reihe 4 (1903) $252-268$.

[Wit] E. Witten: Quantum field theory, Grassmannians, and algebraic curves, Commun. Math. Phys. 113 (1988) 529-600. 